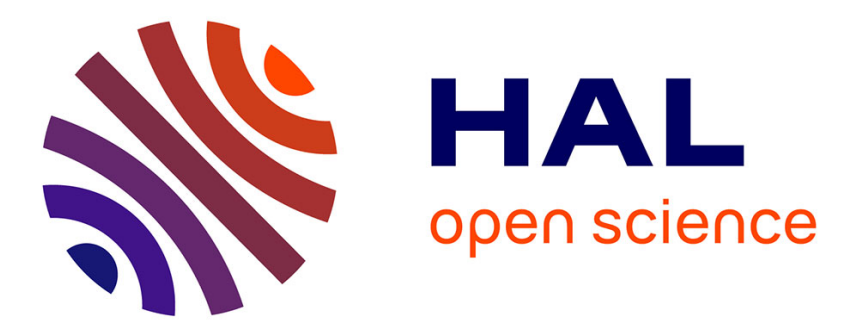

\title{
Statistical modelling of radiative transfer within non-Beerian effective phases of macroporous media Jean Taine, Franck Enguehard
}

\section{To cite this version:}

Jean Taine, Franck Enguehard. Statistical modelling of radiative transfer within non-Beerian effective phases of macroporous media. International Journal of Thermal Sciences, 2019, 139, pp.61-78. 10.1016/j.ijthermalsci.2019.01.012 . hal-02326969

\section{HAL Id: hal-02326969 https://hal.science/hal-02326969}

Submitted on 22 Oct 2021

HAL is a multi-disciplinary open access archive for the deposit and dissemination of scientific research documents, whether they are published or not. The documents may come from teaching and research institutions in France or abroad, or from public or private research centers.
L'archive ouverte pluridisciplinaire HAL, est destinée au dépôt et à la diffusion de documents scientifiques de niveau recherche, publiés ou non, émanant des établissements d'enseignement et de recherche français ou étrangers, des laboratoires publics ou privés.

\section{다)(1) $(5$}

Distributed under a Creative Commons Attribution - NonCommercial| 4.0 International 


\title{
Statistical modelling of radiative transfer within non-Beerian effective phases of macroporous media
}

\author{
Jean Taine ${ }^{* a}$ and Franck Enguehard ${ }^{b}$ \\ ${ }^{a}$ Laboratoire d'Énergétique Moléculaire et Macroscopique, Combustion, \\ UPR 288 du CNRS, CentraleSupélec, Université Paris-Saclay \\ Bât. Eiffel, rue Joliot Curie, 91192 Gif-sur-Yvette Cedex, France \\ ${ }^{b}$ Institut Pprime, CNRS, Université de Poitiers ISAE-ENSMA \\ 86962, Futuroscope, Chasseneuil du Poitou, France \\ *correspondance author: jean.taine@centralesupelec.fr
}

Keywords: Non-Beerian porous medium, Statistical characterisation, Correlated emission source, Correlated scattering source, Generalised Radiative Transfer Equation, Radiative Fourier Law.

\begin{tabular}{ll}
\multicolumn{2}{c}{ List of Symbols } \\
Roman Symbols \\
$A$ & Interfacial area per unit volume \\
$B$ & Generalised extinction coefficient \\
$g$ & Scattering asymmetry factor \\
$G_{\text {ext }}$ & Extinction cumulative distribution function \\
$k, k_{j m}$ & Conductivity, conductivity tensor \\
$K$ & Generalised absorption coefficient at equilibrium \\
$I$ & Radiation intensity \\
$\mathcal{L}$ & Functional \\
$m$ & Real phase material \\
$n$ & Refractive index \\
$\mathbf{n}$ & Normal unit vector \\
$p$ & Scattering phase function \\
$\mathrm{P}$ & Power per unit volume of the whole porous medium \\
$P$ & Cumulative probability \\
$\mathcal{P}$ & Power \\
$\mathbf{q}$ & Flux vector \\
$\mathbf{r},(\mathbf{R})$ & Coordinate, (at macroscale)
\end{tabular}




\begin{tabular}{|c|c|}
\hline$s$ & Curvilinear abscissa \\
\hline$S$ & Radiation source term \\
\hline $\mathbf{u}$ & Direction unit vector \\
\hline$V$ & Volume \\
\hline$w$ & Distance between source and extinction points \\
\hline$x_{j}$ & Tensorial vector coordinate \\
\hline \multicolumn{2}{|c|}{ Greek Symbols } \\
\hline$\alpha$ & Real or effective phase \\
\hline$\beta$ & Extinction coefficient \\
\hline$\gamma$ & Real or effective phase \\
\hline$\delta$ & Scale such that a phase is optically thick \\
\hline$\delta(-)$ & Kronecker distribution \\
\hline$\eta$ & Criterion \\
\hline$\kappa$ & Absorption coefficient \\
\hline$\mu, \mu_{s c}$ & Incidence, Scattering angle cosine \\
\hline$\nu$ & Radiation frequency \\
\hline$\Pi$ & Volume fraction \\
\hline$\rho$ & Reflectivity \\
\hline$\sigma$ & Scattering coefficient \\
\hline$\Sigma$ & Generalised scattering coefficient at equilibrium \\
\hline$\tau$ & transmissivity \\
\hline$\omega$ & Albedo \\
\hline$\Omega$ & Solid angle \\
\hline \multicolumn{2}{|c|}{ Superscripts } \\
\hline$B$ & Beerian \\
\hline$i$ & Incident \\
\hline ot & Optically thin limit \\
\hline$r$ & Reflected \\
\hline$R$ & Radiative \\
\hline$S,(S)$ & $\begin{array}{l}\text { Issued from an interfacial source, an interfacial } \\
\text { or volume source }\end{array}$ \\
\hline$t$ & Transparent real phase \\
\hline $\operatorname{tr}$ & Transmitted \\
\hline " & Bidirectional \\
\hline
\end{tabular}




$\begin{array}{ll}+ & \text { Normalised quantity } \\ { }^{+} & \text {At Ideal Thermal Equilibrium } \\ { }^{(0)},(1) & \text { At zero, first perturbation order }\end{array}$

\section{Subscripts}

$\begin{array}{ll}a & \text { Absorption } \\ b & \text { Porous medium boundary } \\ e & \text { Emission } \\ \text { eff } & \text { Effective } \\ \text { ext } & \text { Extinction } \\ i & \text { Interfacial } \\ \text { sc } & \text { Scattering } \\ \text { Acronyms } & \\ \text { DOOS } & \text { Dispersed Overlapping Opaque Spheres } \\ \text { DOTS } & \text { Dispersed Overlapping Transparent Spheres } \\ \text { GRTE } & \text { Generalised Radiative Transfer Equation } \\ \text { Kn } & \text { Knudsen number } \\ \text { OT } & \text { Opaque and Transparent phases } \\ \text { OST } & \text { Opaque and Semi-Transparent phases } \\ \text { RTE } & \text { Radiative Transfer Equation } \\ \text { STT } & \text { Semi-Transparent and Transparent phases } \\ \text { ST2 } & \text { Two Semi-Transparent phases }\end{array}$

\section{Abstract}

A purely statistical modelling of radiative transfer is applied to statistically anisotropic and sometimes non-homogeneous macroporous media, in which the geometrical optics laws are assumed to be valid. It is focused on the physical features of media characterised, after statistical homogenisation, by non-Beerian effective phases such that the extinction law is not exponential. Approaches that have been developed for media with Opaque and Transparent phases (OT) are generalised to media with Opaque and Semi-Transparent phases (OST), Semi-Transparent and Transparent phases (STT) and two Semi-Transparent phases (ST2). Many assumptions of previous papers are justified or invalidated. Limitations of the models are introduced. 
Non-Beerian effective phases are exhaustively characterised by accurate radiative statistical functions: Extinction cumulative distribution functions, scattering cumulative probabilities and general scattering phase functions. Key studied phenomena are the correlations that occur in radiative transfer: i) Between transmission, from source points to interfacial points, and interfacial extinction; ii) In OT and OST cases, between interfacial emission, the following transmission and interfacial extinction; iii) In the case of non-diffuse reflection or transmission law, between an interfacial incident intensity and an interfacial scattered intensity, and between this scattered intensity and the following transmission and extinction.

In the case of a diffuse reflection or transmission law, the two first types of correlations are taken into account within a non-Beerian effective phase by a unique Generalised Radiative Transfer Equation (GRTE) that is expressed in terms of the radiative statistical functions and also includes the boundary conditions. The GTREs associated with OT, OST, STT and ST2 media are detailed. In the case of a non-diffuse reflection or transmission law, the three types of correlations are taken into account for a non-Beerian effective phase by specific GRTEs. These GRTEs are associated with the successive elementary paths from emission to final absorption after multiple scattering events.

Under specific validity conditions, the GRTE of a homogeneous effective phase of an OT medium degenerates into a classical RTE and a radiative Fourier law can be applied within this effective phase. In the case of an OST or a ST2 medium, this model can only be applied if the homogeneous effective phases are characterised by the same temperature field. A radiative Fourier law is never valid for homogeneous effective phases of a STT medium or for a non-homogeneous effective phase of any type.

A non-homogeneous effective phase, with strong volume fraction gradients, is characterised by radiative statistical functions that also depend on the coordinates of the source points. All the previous models of radiative properties are generalised by using a global transmissivity, product of the volume fraction at the source point by the transmissivity deduced from the extinction cumulative distribution function.

Finally, the spatial limitations of the use of a GRTE or a radiative Fourier law are 
enlightened from a comparison of all the spatial scales involved in the characterisation of the radiative statistical functions with the thickness of the radiative boundary layer of the porous medium and with the spatial resolution of the temperature field.

\section{Introduction}

Macroporous media at high temperature are involved in many technologies of energy (hydrocarbon reforming, solar absorbers, safety of a core of a nuclear reactor, waste combustion, insulators for extreme thermal conditions, etc.). An accurate modelling of radiative transfer has to be achieved in these applications. Radiation is even preponderant in some cases. The present study is devoted to radiative transfer within macroporous media such that the typical radiation wavelength is small compared to the smallest considered spatial scale of the medium. The geometrical optics laws are then assumed to be valid. More specifcally, this work is focused on effective phases, obtained after homogenisation, that exhibit a non-Beerian behaviour, i.e. that are not characterised by an exponential extinction law.

In a recent past, the characterisation of the radiative properties of porous media was generally based on parameter identification techniques. In these approaches, the porous medium was globally homogenised by a one-phase model and this effective phase was always assumed to be Beerian, i.e. characterised by an exponential extinction law. A review of these methods is given in Ref.[1] and many practical developments are gathered in a textbook[2].

In the two last decades, the development of advanced tomography techniques has allowed the morphology of most macroporous media to be well known and the radiative properties of these media to be accurately characterised, in particular by a multi-phase approach. Two families of models have mainly been developed in parallel: On the one hand, models that are based on the spatial averaging theorem and, on the other one, purely statistical models.

Consalvi et al.[3] have first applied to radiative transfer within divided media an approach similar to the volume averaged method of Whitaker and Quintard[4-7], which is based on the spatial averaging theorem. Other approaches based on this 
theorem have been developed: i) For statistically isotropic porous media by Lipinski et al.[8, 9], Randrianalisoa et al.[10-12], Coquard at al.[13, 14], and Gusarov [15-18]. In many cases, an extinction coefficient is based on an extinction mean free path that is determined from a Monte Carlo approach, without using in the model the complete extinction distribution function; ii) For statistically anisotropic media by Gusarov[19].

The spatial averaging theorem is applied to the two coupled radiative transfer equations associated with the real phases. Two scales are then considered[8]: i) The continuous scale of these equations; ii) The discrete scale of the final results. Effective scattering, absorption and extinction coefficients and effective scattering phase functions, which take into account the interfacial effects, are obtained at the discrete scale. The final coefficients are the sum of the intrinsic coefficients and of the corresponding effective ones. The final phase functions are combinations of intrinsic and effective phase functions. Two coupled effective Beerian phases are finally obtained. This model has been successfully applied to different media that verify, at least approximately, the scale separation assumption: Indeed, a validity condition of the spatial averaging theorem is that the continuous and discrete scales are not of the same order of magnitude[4-7]. But strongly non-Beerian effective phases are generally characterised by continuous and discrete scales of comparable orders of magnitude, due to long-range interactions between interfaces. Cite, for instance: i) Regular arrangement of rods with void alleys (see Fig.2); ii) Absorbing long fibres of a felt (see Sec.5.3). Models based on the spatial averaging theorem are questionable in these conditions.

The developments of the $X$ and $\gamma$ tomography techniques[20-25] and of the power of the computers have also allowed an effective phase of a macroporous medium to be accurately characterised by radiative statistical functions: Extinction cumulative distribution functions, scattering cumulative probabilities and general scattering phase functions. The first works have been simultaneously achieved by Tancrez[26] and Petrasch et al.[27], followed by many other authors[20-22, 25, 28-31]. Strongly nonBeerian behaviours have been observed with a high degree of accuracy[25, 29, 31, 32]. As this statistical characterisation of the radiative properties was a new approach, 
many assumptions have been made without justification[20, 25-27, 29, 33]. Moreover, the limitations of the models derived from these assumptions have generally not been enlightened. An ad hoc model related to interfacial emission[25, 32, 34], now appears to be erroneous for a non-Beerian effective phase.

The present theoretical paper deals with a purely statistical modelling of radiative transfer within non-Beerian effective phases of macroporous media, which are in most cases statistically anisotropic and sometimes non-homogeneous. Works that have mainly been achieved for media with Opaque and Transparent phases (OT) are generalised to media with Opaque and Semi-Transparent phases (OST), SemiTransparent and Transparent phases (STT) and two Semi-Transparent phases (ST2). Specific physical phenomena that are involved in these different types of media are enlightened in this original study. Assumptions made in previous papers are then justified or invalidated and the limitations of the models are clarified. Moreover, all these developments are achieved in order to be easily applied in radiative transfer with a controlled spatial resolution, by using a Generalised Radiative Transfer Equation (GRTE) in a general case or, under some conditions, a radiative Fourier law. The key theoretical features of non-Beerian effective phases, which are linked to the homogenisation of the interfacial extinction, are presented with a minimum use of equations in Sec.2 that is focused on the physical understanding of these phenomena. The strong correlations that occur within non-Beerian effective phases between interfacial emission, transmission and extinction and possibly between the previous phenomena and the interfacial scattering source terms are enlightened and discussed. The specific conditions of a Beerian behaviour are also stated.

Sections 3-6 deal with the practical application of the theoretical model of Sec.2 to OT, OST, STT and ST2 media. Section 3 is devoted to an original characterisation of the radiative statistical functions associated with emission and scattering interfacial sources, and to the modelling of the correlations discussed in Sec.2. Generalised Radiative Transfer Equations (GRTEs) associated with OT, OST, STT and ST2 media are expressed in Sec.4 by taking into account the correlations between interfacial phenomena defined in Sec.2 and expressed in Sec.3. The validity of the 
radiative Fourier law, applied to an effective phase or to the whole porous medium, is discussed for OT, OST, STT and ST2 media. When it is valid, a radiative conductivity tensor is directly determined from the radiative statistical functions. The spatial limitations of the different models that are based on the statistical method are enlightened in Sec.6, from a comparison of all the spatial scales involved in the characterisation of the radiative statistical functions with the scale of the optically thick radiative boundary layer and with the spatial resolution of the temperature field.

General conclusions are finally drawn.

\section{Theoretical model}

This Section deals with an original complete presentation of the key theoretical features of radiative transfer within non-Beerian effective phases of a macroporous medium, by comparison with the case of Beerian media. Published partial results are synthetised, corrected and mainly generalised

In the statistical approach, two effective phases $\alpha$ and $\gamma$ are assumed to be present at any point $M^{\prime}\left(\mathbf{r}^{\prime}\right)$ of a physical volume element $\mathrm{d} V$ of the whole porous medium with presence probabilities $\Pi_{\alpha}$ and $\Pi_{\gamma}$, which are the corresponding volume fractions. An interfacial element is also statistically assumed to be present at any point, as it is commonly done for small particles within a particle-gas mixture. The radiative exchange between interfacial elements occurs within a continuous semi-transparent effective phase, even if the corresponding real phase is transparent. After homogenisation, the possible non-Beerian behaviour of an effective phase is due to this interfacial exchange, which often corresponds to a long-range interaction.

A non-Beerian effective phase is characterised by a non-exponential transmissivity $\tau_{\nu \alpha}\left(\mathbf{u}, s, s^{\prime}-s\right)$ between two points $M$ and $M^{\prime}$, of abscissa $s$ and $s^{\prime}$ along an axis of direction u. Consequently, extinction, scattering and absorption coefficients have no physical meaning. If the effective phase is homogeneous, characterised by a uniform volume fraction $\Pi_{\alpha}$, the transmissivity $\tau_{\nu \alpha}\left(\mathbf{u}, s^{\prime}-s\right)$ is independent of the position $M(s)$ of the source. This assumption will be implicitly made in the following with the exception of Secs.3.4 and 6.2. 
When interfacial extinction is not ruled by an exponential law, the knowledge of the radiative intensity at a point is not sufficient for characterising the variation of this intensity at this point within a non-Beerian effective phase:

i) Correlations occur in the OT, OST, STT and ST2 cases between transmission up to an interfacial point and interfacial extinction at this point, as shown in Sec.2.1;

ii) In OT and OST cases, correlations also occur between interfacial emission, the following transmission up to an interface and interfacial extinction (Sec.2.2);

iii) If the real phases are characterised by a non-diffuse interfacial transmission or reflection law, such as a Fresnel law, the intensity that is scattered by the interfaces at a point within an effective phase is also strongly correlated with the incident intensity at this point, as shown in Sec.2.3. On the contrary, such a correlation does not occur in the case of a diffuse transmission or reflection law that is independent on both the incidence and final directions. A necessary and sufficient condition for an effective phase to be Beerian is also stated in this Section.

The bases of a statistical modelling of radiative transfer within an effective phase that takes into account all the previously defined correlations are introduced in Sec.2.4. Finally, Section 2.5 deals with a critical study of previous statistical works that is based on the discussions of the present Section.

\subsection{Correlation between interfacial transmission and extinction}

Consider first, within an effective phase, a radiative source term $S_{\nu}(\mathbf{u}, \mathbf{s})$ due to: i) Either emission or scattering by a semi-transparent real phase; ii) Or interfacial scattering that is associated with a diffuse transmission and reflection law within the real phases. $S_{\nu}(\mathbf{u}, \mathbf{s})$ is issued from an interval $[s, s+d \mathbf{s}]$ of an axis of direction $\mathbf{u}$. As shown in the following Sections, there is no correlation in the previously defined

conditions between $S_{\nu}(\mathbf{u}, \mathbf{s})$ and the following transmissivity $\tau_{\nu}\left(\mathbf{u}, s^{\prime}-s\right)$ from $s$ to $s^{\prime}$, even for a non-Beerian effective phase.

If the effective phase is Beerian, the variation within the interval $\left[s^{\prime}, s^{\prime}+d \mathrm{~s}^{\prime}\right]$ of the transmissivity from $s$ to $s^{\prime}$ is equal to $\tau_{\nu \alpha}\left(\mathbf{u}, s^{\prime}-s\right) \beta_{\nu}(\mathbf{u}) \mathrm{d} s^{\prime}$, where $\beta_{\nu}(\mathbf{u})$ is an extinction coefficient that is independent of $s^{\prime}-s$ and $\tau_{\nu \alpha}\left(\mathbf{u}, s^{\prime}-s\right)$ is equal to $\exp \left[-\beta_{\nu}(\mathbf{u})\left(s^{\prime}-s\right)\right]$. The variation of the global intensity due to extinction at $s^{\prime}$ 
simply writes in these conditions

$$
\mathrm{d} I_{\nu \alpha}^{e x t}\left(\mathbf{u}, s^{\prime}\right)=-\beta_{\nu}(\mathbf{u}) \mathrm{d} s^{\prime} \int_{0}^{s^{\prime}} S_{\nu}(\mathbf{u}, s) \exp \left[-\beta_{\nu}(\mathbf{u})\left(s^{\prime}-s\right)\right] \mathrm{d} s=-\beta_{\nu}(\mathbf{u}) \mathrm{d} s^{\prime} I_{\nu \alpha}\left(\mathbf{u}, s^{\prime}\right) .
$$

The global intensity $I_{\nu \alpha}\left(\mathbf{u}, s^{\prime}\right)$ then synthetises all the information required for determining $\mathrm{d} I_{\nu \alpha}^{e x t}\left(\mathbf{u}, s^{\prime}\right)$, which drastically simplifies the model. There is no correlation between transmission from $s$ to $s^{\prime}$ characterised by $\tau_{\nu \alpha}\left(\mathbf{u}, s^{\prime}-s\right)$ and extinction between $s^{\prime}$ and $s^{\prime}+\mathrm{d} s^{\prime}$ characterised by $\beta_{\nu}(\mathbf{u}) \mathrm{d} s^{\prime}$.

But, for a non-Beerian effective phase that is characterised by a non-exponential extinction law, extinction coefficients, and consequently absorption and scattering coefficients, can not be defined. The variation of the transmissivity within $\left[s^{\prime}, s^{\prime}+\mathrm{d} s^{\prime}\right]$ can be expressed as the product of the transmissivity $\tau_{\nu \alpha}\left(\mathbf{u}, s^{\prime}-s\right)$ by a function that characterises extinction between $s$ and $s^{\prime}$ and also depends on the distance $s^{\prime}-s$. There is a strong correlation between transmission from $s$ to $s^{\prime}$ and extinction and consequently scattering or absorption within $\left[s^{\prime}, s^{\prime}+\mathrm{d} s^{\prime}\right]$. The variation of the global intensity due to extinction between $s^{\prime}$ and $s^{\prime}+\mathrm{d} s^{\prime}$ then writes

$$
\mathrm{d} I_{\nu \alpha}^{e x t}\left(\mathbf{u}, s^{\prime}\right)=\mathrm{d} s^{\prime} \int_{0}^{s^{\prime}} S_{\nu}(\mathbf{u}, s) \frac{\mathrm{d} \tau_{\nu \alpha}\left(\mathbf{u}, s^{\prime}-s\right)}{\mathrm{d} s^{\prime}} \mathrm{d} s
$$

It can not be expressed as a function of this intensity and is only obtained by summing the contributions of all the sources. There is a memory effect of all the radiation sources.

\subsection{Correlated interfacial emission sources}

According to the discussion of Sec.2.1, interfacial emission in OT and OST cases can not be expressed by using an absorption coefficient, as commonly done for a Beerian semi-transparent medium.

This difficulty is solved by using the reciprocity theorem, which is issued from the detailed balance principle[35, 36] in statistical physics. This theorem states that interfacial emission between $s$ and $s+\mathrm{d} s$ in the direction $\mathbf{u}$ can be expressed from interfacial absorption between $s+\mathrm{d} s$ and $s$ in the reverse direction $-\mathbf{u}$. As interfa- 
cial absorption is strongly correlated with transmission from $s^{\prime}$ to $s$, the interfacial emission source is strongly correlated with transmission from $s$ to $s^{\prime}$ and interfacial extinction within $\left[s^{\prime}, s^{\prime}+\mathrm{d} s^{\prime}\right]$. The corresponding equations are developed in Sec.3.2.2 in the case of a diffuse reflection law. The much more complex approach associated with a non-diffuse reflection law is exposed in Sec.3.3.

\subsection{Correlated and non-correlated interfacial scattering sources}

Within an effective phase $\alpha$ of an OT or OST medium, the scattering source term in a given direction $\mathbf{u}$ associated with an incident radiation in a direction $\mathbf{u}_{\mathbf{1}}$ depends on the reflection law at the interfaces of the real phase. Similarly, for a STT or ST2 medium, the scattering source terms within the effective phases $\alpha$ and $\gamma$ in the direction $\mathbf{u}$ depend on the interfacial transmission and reflection laws of the real phases. For the sake of simplicity, we only consider here the first case and assume that the interfacial reflection law simply depends on the incidence angle cosine $\mu_{1}$ at an impact point $M^{\prime}$. The results associated with a STT or ST2 medium can easily be deduced from all the results obtained for an OT or OST medium.

The rays that are issued from points $M(s)$ in an elementary solid angle $\mathrm{d} \Omega_{1}$ around the direction $\mathbf{u}_{1}$ and impact interfaces at points $M^{\prime}\left(s^{\prime}\right)$ are characterised by the distribution function $f_{\alpha}\left(s^{\prime}-s, \mathbf{u}_{1}\right)$ of the distances $M M^{\prime}$ and by the distribution function $f_{\alpha}\left(\mu_{1}, \mathbf{u}_{1}\right)$ of $\mu_{1}$. Three cases occur and are considered in the following:

i) $\mu_{1}$ and $s^{\prime}-s$ are statistically independent, whatever the interfacial reflection law. This case corresponds to a Beerian effective phase, as shown in Sec.2.3.1;

ii) Interfacial reflection within the real phases is diffuse, independent of the initial and final directions. This commonly considered case is discussed in Sec.2.3.2;

iii) Reflection is not diffuse and $\mu_{1}$ and $s^{\prime}-s$ are not statistically independent, general case discussed in Sec.2.3.3.

\subsubsection{Beerian effective phase}

Assume first that, within the real phase $\alpha$ of an OT or OST medium, the incidence angle cosines $\mu_{1}$ at the impact points $M^{\prime}$ of the rays belonging to an elementary solid angle around the direction $\mathbf{u}_{1}$ are statistically independent of the distances 
$M M^{\prime}=s^{\prime}-s$ from the source points $M(s)$. The internal scattering directions within the effective phase $\alpha$, which depend on the distribution function $f_{\alpha}\left(\mu_{1}, \mathbf{u}_{1}\right)$ of $\mu_{1}$ through the reflection law, are then independent of $s^{\prime}-s$.

Within the effective phase $\alpha$, there is then no correlation between the transmissivity from $M$ to $M^{\prime}$ and the internally scattered intensity at the point $M^{\prime}$ in $\mathrm{d} \Omega$, around the direction $\mathbf{u}$. The global variation of the incident intensity $I_{\nu \alpha}\left(\mathbf{u}_{1}, s^{\prime}\right)$ in the direction $\mathbf{u}_{\mathbf{1}}$ at the point $M^{\prime}\left(s^{\prime}\right)$ that is due to internal scattering in all directions is then proportional to $I_{\nu \alpha}\left(\mathbf{u}_{1}, s^{\prime}\right)$ and, consequently, to an internal scattering coefficient $\sigma_{\nu \alpha \alpha}\left(\mathbf{u}_{1}\right)$. The scattering source term within an elementary solid angle $\mathrm{d} \Omega$ is then characterised by a unique phase functions $p_{\nu \alpha \alpha}\left(\mathbf{u}_{1}, \mathbf{u}\right)$, which depends both on the reflection law and the morphology of the medium. The variation of the intensity at the point $M^{\prime}\left(s^{\prime}\right)$ due to absorption is also proportional to $I_{\nu \alpha}\left(\mathbf{u}_{1}, s^{\prime}\right)$ and to an absorption coefficient $\kappa_{\nu \alpha}\left(\mathbf{u}_{1}\right)$.

The statistical independence of $s^{\prime}-s$ and $\mu_{1}$ is then a sufficient condition of a Beerian behaviour. If, on the contrary, it is assumed that $s^{\prime}-s$ and $\mu_{1}$ are statistically dependent, a strong correlation will occur between the scattered or absorbed intensity and the history of the incident radiation, as shown in Sec.2.3.3: Absorption or scattering coefficients can then not be defined. The statistical independence of $s^{\prime}-s$ and $\mu$ finally is a necessary and sufficient condition of a rigorous Beerian behaviour of an effective phase.

Note that, in the case of a diluted medium (high volume fraction value), there is no strong variation of $s^{\prime}-s$ and the higher is the dilution the weaker is the variation of $s^{\prime}-s$. The correlations between $\mu_{1}$ and $s^{\prime}-s$ are then weak independently of the geometrical configuration and the medium is then practically Beerian.

This condition is illustrated in Fig.1[26, 34] by the opposite behaviours of two models of statistically isotropic and homogeneous foams:

i) Dispersed Overlapping Opaque Spheres within a transparent medium (DOOS);

ii) Dispersed Overlapping Transparent Spheres within an opaque medium (DOTS). The logarithm of the transmissivity within the effective phase is plotted vs the optical thickness $\beta^{o t} w$ at the optically thin limit, equal to $A w /(4 \Pi)[26,37]$, where $A$ and $\Pi$ are the specific area per unit volume of the medium and the volume fraction of 


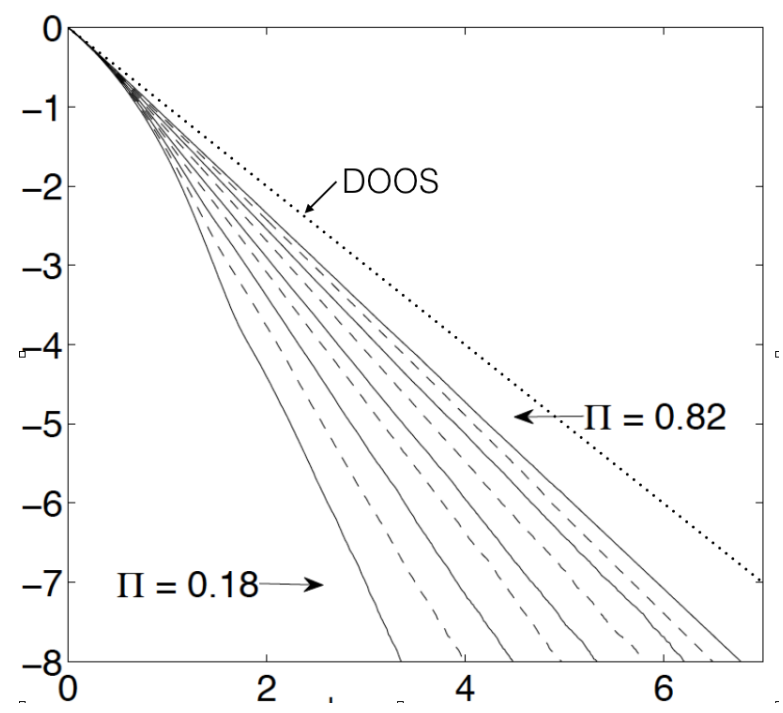

Figure 1: DOOS and DOTS: $\ln [\tau(w)]$ vs $\beta^{o t} w$ for different porosity $\Pi$ values[34] from the model of Ref.[26]; DOOS: universal dotted straight line; DOTS: Curves associated with $\Pi=$ 0,$18 ; 0,26 ; 0,37 ; 0,48 ; 0,56 ; 0,65 ; 0,72 ; 0,78 ; 0,82$ (EM2C/GDF study).

the phase. A curve associated with a Beerian effective phase is then a universal straight line of slope -1 . It is rigorously the case for DOOS: $\beta^{\text {ot }}$ is then valid for all the optical thickness range. On the contrary, the effective phase of DOTS is never exactly Beerian. Whatever the porosity value, $\beta^{o t}$ is valid for the weak optical thicknesses, but another extinction coefficient, which strongly increases when the porosity decreases, characterises the large optical thicknesses. For high porosity values, as previously discussed, a DOTS effective phase can also approximately be considered as Beerian, with an optimal extinction coefficient value close to $\beta^{\text {ot }}$. But it is more and more non-Beerian when the porosity decreases, even though the medium is statistically isotropic.

In fact, for DOTS or within a simple sphere, the chord lengths $s^{\prime}-s$ and the incidence angle cosine value $\mu_{1}$ are strongly correlated: For instance, the values $\mu_{1}=1$ and $s^{\prime}-s=D$, where $D$ is the diameter of a sphere, are totally correlated. The condition of a Beerian behaviour is then not fulfilled. On the contrary, in the case of DOOS, $\mu_{1}$ and $s^{\prime}-s$ are statistically independent.

Another example is given by a statistically axi-symmetrical felt made of overlapping 
infinite semi-transparent cylinders within a transparent phase[31], more specifically defined in Sec.5.3. The medium is statistically isotropic in two dimensions. The effective phase outside the cylinders is strongly Beerian, as in the DOOS case. But the semi-transparent phase is strongly non-Beerian: Indeed, within a cylinder, $\mu_{1}$ is clearly correlated with the chord length $s^{\prime}-s$.

The same results can obviously be obtained for Beerian effective phases of STT and ST2 media.

\subsubsection{Diffuse reflection law}

If the interfacial reflection law is diffuse within the real phase $\alpha$ of an OT or OST medium, the probability that any ray that is incident in $\mathrm{d} \Omega_{1}\left(\mathbf{u}_{1}\right)$ at a given point $M^{\prime}\left(s^{\prime}\right)$ will be reflected in $\mathrm{d} \Omega(\mathbf{u})$ is independent of $\mu_{1}$ and consequently of $s^{\prime}-s$. It only depends on the geometrical configuration, more specifically on the belonging of $\mathrm{d} \Omega$ to the real phase $\alpha$. In these conditions, even for a non-Beerian effective phase $\alpha$, the phase function $p_{\nu \alpha \alpha}\left(\mathbf{u}_{1}, \mathbf{u}\right)$ is unique but not isotropic and only depends on the morphology of the medium. The associated scattered intensity in $\mathrm{d} \Omega(\mathbf{u})$ is proportional, as in a Beerian semi-transparent medium, to the global incident intensity $I_{\nu \alpha}\left(\mathbf{u}_{1}, s^{\prime}\right)$ and to the phase function $p_{\nu \alpha \alpha}\left(\mathbf{u}_{1}, \mathbf{u}\right)$. This property used in Sec.2.1 can be generalised to STT and ST2 media and drastically simplifies the models. Nevertheless, interfacial extinction by scattering or absorption is correlated with the incident radiation in the case of a non-Beerian effective phase.

In the paper, transmission or reflection is assumed to be diffuse for OT, OST, ST and ST2 media with the exceptions of Secs.2.3.3 and 3.3.

\subsubsection{Non-diffuse transmission and reflection laws (Non-Beerian case)}

Within a real phase $\alpha$ of an OT medium, all the rays belonging to an elementary solid angle $\mathrm{d} \Omega_{1}$ around a direction $\mathbf{u}_{1}$ that are issued from interfacial points $M(s)$ and incident over interfacial elements at points $M\left(s^{\prime}\right)$ are characterised by the distri-

bution functions $f_{\alpha}\left(s^{\prime}-s, \mathbf{u}_{1}\right)$ of the distances $M M^{\prime}$ and $f_{\alpha}\left(\mu_{1}, \mathbf{u}_{1}\right)$ of the incidence angle cosine $\mu_{1}$, as shown in the introduction of Sec.2.3. These distribution functions only depend on the morphology of the porous medium. In many cases, $\mu_{1}$ and $s^{\prime}-s$ 
are not statistically independent: For instance, in the extreme case of a medium between two parallel planes, $\mu_{1}$ and $s^{\prime}-s$ are totally correlated. When $\mu_{1}$ and $s^{\prime}-s$ are not statistically independent, the interfacial reflection law is correlated with $s^{\prime}-s$. In these conditions, the scattered intensity in a direction $\mathbf{u}$ at a point $M^{\prime}\left(s^{\prime}\right)$ within the effective phase $\alpha$ is correlated with the transmissivity $\tau_{\alpha \nu}\left(\mathbf{u}_{1}, s^{\prime}-s\right)$.

In the same manner, all the rays that are reflected within the real phase at the interfacial points $M^{\prime}\left(s^{\prime}\right)$ in the elementary solid angle $\mathrm{d} \Omega(\mathbf{u})$ and impact another interfacial elements at the points $M^{\prime \prime}\left(s^{\prime \prime}\right)$ are characterised by the distribution functions $f_{\alpha}\left(s^{\prime \prime}-s^{\prime}, \mathbf{u}\right)$ of the distances $s^{\prime \prime}-s^{\prime}$ and $f_{\alpha}(\mu, \mathbf{u})$ of the reflection angle cosine $\mu$, which only depend on the medium morphology. As $\mu_{1}$ and $s^{\prime}-s, \mu$ and $s^{\prime \prime}-s^{\prime}$ are

not statistically independent. The scattering intensity within the effective phase $\alpha$ at $M^{\prime}\left(s^{\prime}\right)$ in the direction $\mathbf{u}$ is also correlated with the transmissivity $\tau_{\alpha \nu}\left(\mathbf{u}, s^{\prime \prime}-s^{\prime}\right)$ from $s^{\prime}$ to $s^{\prime \prime}$.

Consequently, after multiple scattering events, the scattered intensity at a point $M^{\prime}$ of the effective phase in a given direction is correlated with all the successive transmission and scattering phenomena from the non-correlated emission point within a real semi-transparent phase, in OST, STT or ST2 case, or from the correlated interfacial emission point, in OT or OST case, up to the scattering point $M^{\prime}$. This complex case is developed in Sec.3.3.

\subsection{Radiative transfer modelling}

Any model of radiative transfer is based on the determination of the radiative flux vector $\mathbf{q}_{\alpha}^{R}\left(\mathbf{r}^{\prime}\right)$ and the radiative power per unit volume $P_{\alpha}^{R}\left(\mathbf{r}^{\prime}\right)$ at a point $M^{\prime}\left(\mathbf{r}^{\prime}\right)$ of an effective phase $\alpha$, of abscissa $s^{\prime}$ along an axis of direction $\mathbf{u}$, i.e.

$$
\begin{aligned}
\mathbf{q}_{\alpha}^{R}\left(\mathbf{r}^{\prime}\right) & =\int_{0}^{\infty} \int_{0}^{4 \pi} I_{\nu \alpha}\left(\mathbf{u}, \mathbf{r}^{\prime}\right) \mathbf{u} \mathrm{d} \Omega_{\alpha}(\mathbf{u}) \mathrm{d} \nu \\
P_{\alpha}^{R}\left(\mathbf{r}^{\prime}\right) & =-\operatorname{div}\left[\mathbf{q}_{\alpha}^{R}\left(\mathbf{r}^{\prime}\right)\right]=-\int_{0}^{\infty} \int_{0}^{4 \pi} \frac{\mathrm{d} I_{\nu \alpha}\left(\mathbf{u}, s^{\prime}\right)}{\mathrm{d} s^{\prime}(\mathbf{u})} \mathrm{d} \Omega_{\alpha}(\mathbf{u}) \mathrm{d} \nu .
\end{aligned}
$$

The intensity $I_{\nu \alpha}, \mathbf{q}_{\alpha}^{R}$ and $P_{\alpha}^{R}$ are defined by unit volume $\mathrm{d} V$ of the whole porous medium, i.e. are proportional to the the phase volume fraction $\Pi_{\alpha}$. 
As detailed in Secs. 2.1-2.3, the key difficulty is to express for a non-Beerian effective phase $\mathrm{d} I_{\nu \alpha}\left(\mathbf{u}, s^{\prime}\right)$, the variation of the intensity between $s^{\prime}$ and $s^{\prime}+\mathrm{d} s^{\prime}$, due on the one hand to extinction by scattering and absorption and on the other one to emission and scattering sources. As extinction, scattering and absorption coefficients have then no physical meaning for interfacial phenomena, a classical Radiative Transfer Equation (RTE) based on such quantities is not valid. A Generalised Radiative Transfer Equation (GRTE) is then directly expressed in terms of the extinction cumulated distribution function, scattering and absorption cumulated probabilities and general phase functions (see Sec.4). When some restrictive conditions are fulfilled, simple radiative Fourier laws are directly deduced from the statistical radiative properties, in OT, OST and ST2 cases, as detailed in Sec.5.

Different models of temperature fields can be implemented:

i) If the effective phases are at equilibrium at temperature $T$ within $\mathrm{d} V, P^{R}[T(\mathbf{r}), \mathbf{r}]$ is a source term of the unique energy equation, similar to the one of a reactive medium; ii) In the case of an OT or an OST medium, if the interfacial temperature is equal to the temperature of the solid opaque phase $T_{s}(\mathbf{r})$, which differs from the one of the gaseous (semi-)transparent phase $T_{g}(\mathbf{r})$, the radiative power within the energy equation of the solid phase writes: $P_{s}^{R}\left[T_{s}(\mathbf{r}), \mathbf{r}\right]=P^{R}\left[T_{s}(\mathbf{r}), \mathbf{r}\right] / \Pi_{s}$, where $\Pi_{s}$ is the solid phase volume fraction.

iii) If, in a more advanced approach, the temperatures of the two effective phases $T_{g}(\mathbf{r})$ and $T_{s}(\mathbf{r})$ and of the interfaces $T_{i}(\mathbf{r})$ of an OT or OST medium differ, the interfacial flux writes: $\varphi^{R}=P^{R}\left[T_{i}(\mathbf{r}), \mathbf{r}\right] / A$, where $A$ is the porous medium specific area;

iv) For a medium with a semi-transparent solid phase and a transparent gaseous one (STT) the radiative power $P_{s}^{R}\left[T_{s}(\mathbf{r}), \mathbf{r}\right]$ is simply applied to the solid phase energy equation;

v) In the case of a medium with two semi-transparent phases (ST2) $P_{s}^{R}\left[T_{s}(\mathbf{r}), \mathbf{r}\right]$ and $P_{g}^{R}\left[T_{g}(\mathbf{r}), \mathbf{r}\right]$ are applied to the solid phase and gaseous phase energy equations, respectively.

Unless otherwise specified, the results of the models iii), iv) and v) are presented in the following. It is easy to deduce the results of the models i) and ii) from the 
previous ones.

A GRTE, which is directly expressed in terms of cumulative distribution functions, is easily solved by a statistical Monte Carlo radiative transfer model, which leads by taking into account the radiative boundary conditions to the determination of $I_{\nu \alpha}\left(\mathbf{u}, \mathbf{r}^{\prime}\right)$ and $\frac{\mathrm{d} I_{\nu \alpha}\left(\mathbf{u}, s^{\prime}\right)}{\mathrm{d} s^{\prime}(\mathbf{u})}$, and consequently of the fields of radiative flux $\mathbf{q}_{\alpha}^{R}\left(\mathbf{r}^{\prime}\right)$ and power per unit volume $P_{\alpha}^{R}\left(\mathbf{r}^{\prime}\right)$ by Eqs 3 and 4 .

As the implementation of the Monte Carlo radiative transfer model does not present any specific originality, this transfer method is not detailed in the paper.

\subsection{Critical comments on previous works}

The models based on the statistical approach have been built step by step from $2004[26,27]$ without a full knowledge of the theoretical bases associated with a nonBeerian effective phase. Some first approaches are here discussed in the light of the developments of Secs.2.1-2.4.

In first works[20-22, 26-29], a Beerian behaviour of an effective phase has been assumed. The numerical determination of the transmission field $\tau_{\nu \alpha}\left(\mathbf{u}, s^{\prime}-s\right)$ from any volume source point to any interfacial point of the effective phase has allowed this Beerian behaviour to be numerically checked. Insofar as an effective phase is considered, at least approximately, as Beerian by taking into account an accuracy criterion, scattering, absorption and extinction coefficients can be identified and a general scattering phase function directly built.

But the effective phases of some samples of porous media[25, 29] and of some classical models $[26,29,31]$ clearly present a non-Beerian behaviour. In particular, in case of strong anisotropy an effective phase generally is non-Beerian[25, 29, 31].

In a first attempt for modelling by a statistical approach the radiative transfer within a non-Beerian effective phase, the correlations between transmission and extinction discussed in Sec.2.1 are taken into account in a first formulation of a Generalised Radiative Transfer Equation(GRTE)[34]. But, in this equation that has only been defined in the core of the medium an interfacial scattering source term is assumed to be non-correlated with the incident intensity and interfacial emission has been empirically expressed. This first model has not been implemented. A correct GRTE 
has been numerically implemented for radiative transfer within different strongly non-homogeneous OT media characterised by a diffuse reflection law [30]. It accounts for the radiative boundary conditions and pragmatically for the correlations between interfacial emission, transmission and extinction defined in Sec.2.2. In another work[31] dedicated to a STT medium, a felt of absorbing fibres characterised by Fresnel transmission and reflection laws, all the correlations discussed in Sec.2.3.3 are taken into account and different GRTEs have been associated with the paths between successive scattering events within any effective phase.

A radiative transfer model based on a radiative Fourier law, valid in restrictive conditions such that the medium becomes Beerian at the considered scale, has led to express a radiative conductivity for an isotropic OT medium[34] and to a radiative conductivity tensor for an anisotropic OT medium[25].

The following Sections 3-5 deal with the practical implementation of the theoretical models of the present Section.

\section{Correlated radiative properties of an effective phase}

The characterisation of the extinction, scattering and absorption properties of an effective phase associated with a real transparent propagation phase is directly achieved by statistical numerical approaches. These approaches are, in practice, specific Monte Carlo methods, which strongly differ from Monte Carlo radiative transfer methods. They have extensively been described by many authors[20-22, 25-29] and are not presented here. In these methods, an accurate knowledge of the medium morphology is required. It is issued from tomography data[20-22, 25, $28]$ with a spatial resolution $a$ or is theoretically known[26, 29, 30]. Moreover, the interfacial radiative properties have to be known at a scale smaller than $a$. The laws of geometrical optics are assumed to be valid: $a$ has to be larger than the typical radiation wavelength.

Section 3.1 is an original presentation of the radiative statistical functions that characterise of a non-Beerian effective phase: Specific functions are associated, on one hand, with interfacial radiation sources and, in the other hand, with volume sources. In the case of diffuse interfacial transmission and reflection laws, an equation which 
links the two types of functions generalises recent results[38]. The borderline case of a Beerian effective phase is finally discussed.

Section 3.2 deals with the specificities of a non-Beerian effective phase associated with a real phase with diffuse transmission and reflection laws. The interfacial noncorrelated scattering source terms and the correlated interfacial emission source term are expressed, as results of the physical discussions of Sec.2.3.2 and 2.2 respectively. The obtained equations generalise the results of Zarrouati et al.[30], obtained in specific conditions.

The case of a non-Beerian effective phase associated with a real phase characterised by non-diffuse transmission and reflection laws, in particular Fresnel laws, which has been recently studied by Dauvois et al.[31] for a STT medium is generalised in Sec.3.3 to other media.

The principles of the modelling of a non-homogeneous effective phase, recently developed by Zarrouati et al.[30], are exposed in Sec.3.4. This approach is the key of future advanced accurate models as developed in Sec.6.

\subsection{General statistical radiative properties}

Within a real phase, the emission sources are interfacial (OT and OST cases) or belong to the phase volume (OST, ST2 and STT cases); The scattering sources belong to the phase volume (OST, STT and ST2 cases) or are interfacial (all cases). In the following, any quantity associated with an interfacial source presents a superscript ${ }^{S}$, unlike the case of a quantity associated with a volume source. A quantity with the superscript ${ }^{(S)}$ is related to a volume source or an interfacial one.

\subsubsection{General definitions}

Extinction within any type of homogeneous effective phase $\alpha$ is characterised by an extinction cumulative distribution function $G_{\text {ext } \nu \alpha}^{(S)}(\mathbf{u}, w)$, which a priori depends on the frequency, on the direction $\mathbf{u}$ and on the distance $w=s^{\prime}-s$ within the real phase between a source point $M(s)$ and the corresponding extinction point $M^{\prime}\left(s^{\prime}\right)$ of an axis of direction $\mathbf{u}$. Physically, $1-G_{\text {ext } \nu \alpha}^{(S)}(\mathbf{u}, w)$ represents the transmissivity $\tau_{\nu \alpha}^{(S)}(\mathbf{u}, w)$ between $M$ and $M^{\prime}$ within the effective phase. If the effective phase $\alpha$ is 
associated with a transparent real phase (OT or STT case), $G_{e x t \alpha}^{S t}(\mathbf{u}, w)$ is also the cumulative distribution function of the chords $M M^{\prime}$ within this last phase, which is independent of $\nu$.

In the following, the superscript ${ }^{t}$ is related to an effective phase associated with a transparent real phase of an OT or STT medium or a virtual one of any medium. An accurate expression of $G_{e x t \nu \alpha}^{t}$ is given in Ref.[20]. The expression of $G_{\text {ext } \nu \alpha}^{S t}$ is similar.

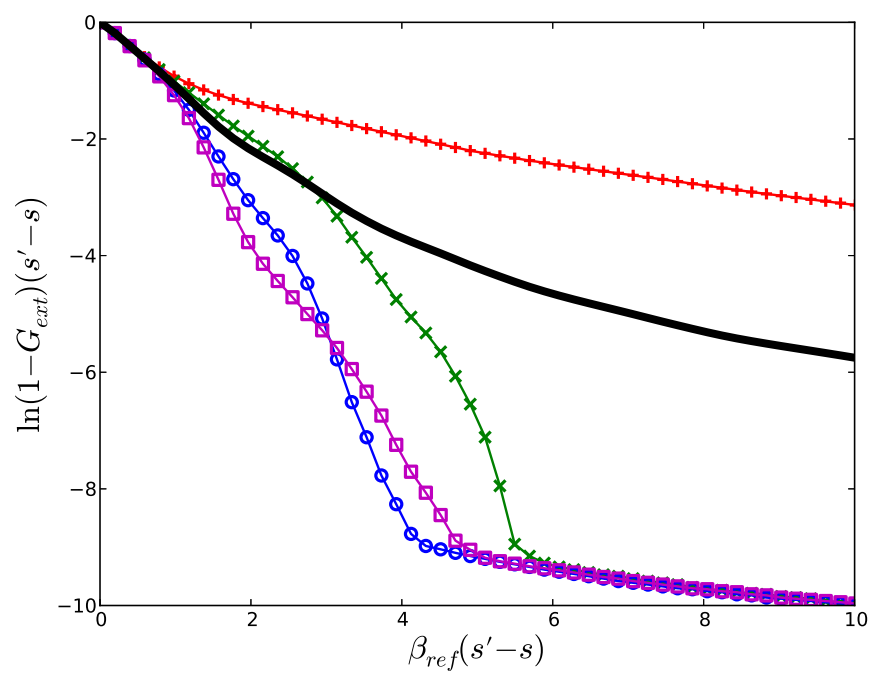

Figure 2: For a degraded cross section of a rod bundle, $\ln \left[1-G_{e x t}^{t}(\theta=\pi / 2, \varphi, w)\right]$ vs $\beta^{o t} w$; From above to below: $\varphi=1.5^{\circ}$ (red crosses); $\varphi=16.5^{\circ}$ (green crosses); $\varphi=31.5^{\circ}$ (blue circles); $\varphi=43.5^{\circ}$ (violet squares); Bold black line associated with $\ln \left[1-G_{e x t}^{t}(\theta=\pi / 2, w)\right]$ averaged over $\varphi$ values[25] (EM2C/IRSN study).

An example of a strongly non-Beerian extinction cumulative distribution functions associated with a strongly anisotropic effective phase is shown in Fig.2: A degraded rod bundle of a nuclear core that has been submitted to the conditions of a severe nuclear accident[25]. The extinction cumulative distribution functions $G_{\text {ext }}^{t}(\theta=\pi / 2, \varphi, w)$ of this OT medium have been determined from the images built from $\gamma$ ray tomography experiments $[25] ; \theta=\pi / 2$ corresponds to a plane perpendicular to the initially intact rods; $\varphi$ is the azimuth within this plane; $\beta^{\text {ot }}$, equal to $A /(\pi \Pi)$ where $A$ is the specific area and $\Pi$ the porosity of the medium, is the 
extinction coefficient at the optically thin limit for a statistically axi-symmetrical configuration[29].

For a Beerian medium, $\ln \left[1-G_{e x t}^{t}(\theta=\pi / 2, \varphi, w)\right]$ would be represented, as for DOOS in Fig.1, by a decreasing straight line of slope -1 , which also occurs in the case of small optical thicknesses. For $\beta^{o t} w$ values that correspond to the semi-transparency range, up to 5 , the behaviour of $\ln \left[1-G_{e x t}^{t}(\theta=\pi / 2, \varphi, w)\right]$ is strongly non-Beerian. Note that the bold black line associated with values of $\ln \left[1-G_{e x t}^{t}(\theta=\pi / 2, w)\right]$ averaged over the azimuth $\varphi$ is much closer to a Beerian behaviour.

A comment related to the modelling of radiative transfer can be deduced from the previous results. Many studies dedicated to anisotropic porous media have been limited to an isotropic characterisation of their radiative properties, which has often led to assume, at least approximately, a Beerian behaviour, as for instance shown in Fig.2. As the radiative flux vector is generally oriented in a main direction, a radiative transfer model that is based on averaged isotropic and Beerian data would lead to erroneous results of radiative transfer by comparison with the results of a model based on anisotropic non-Beerian data.

In OST, STT or ST2 media, extinction, scattering and absorption within the real Beerian semi-transparent phase, characterised by extinction, scattering and absorption coefficients $\beta_{m_{\alpha} \nu}, \sigma_{m_{\alpha} \nu}$ and $\kappa_{m_{\alpha} \nu}$, are statistically independent of the corresponding interfacial phenomena. $G_{\text {ext } \nu \alpha}^{(S)}(\mathbf{u}, w)$ then writes for the effective phase of such a medium

$$
w=s^{\prime}-s, \quad 1-G_{\text {ext } \nu \alpha}^{(S)}(\mathbf{u}, w)=\exp \left(-\beta_{m_{\alpha} \nu} w\right)\left[1-G_{\text {ext } \alpha}^{(S) t}(\mathbf{u}, w)\right]
$$

Interfacial absorption and interfacial internal scattering within an effective phase that is associated with a real transparent phase (OT case) or a virtual one (OST case) are characterised by absorption and internal scattering cumulative probabilities $P_{a \nu \alpha}^{(S) t}(\mathbf{u}, w)$ and $P_{s c \nu \alpha \alpha}^{(S) t}(\mathbf{u}, w)$, respectively. The corresponding extinction cumulative distribution function verifies

$$
G_{\text {ext } \alpha}^{(S) t}(\mathbf{u}, w)=P_{s c \nu \alpha \alpha}^{(S) t}(\mathbf{u}, w)+P_{a \nu \alpha}^{(S) t}(\mathbf{u}, w)
$$


Similarly, interfacial internal and external scattering within an effective phase that is associated with a real transparent phase (STT case) or a virtual one (ST2 case) are characterised by internal and external scattering cumulative probabilities $P_{s c \nu \alpha \alpha}^{(S) t}(\mathbf{u}, w)$ and $P_{s c \nu \alpha \gamma}^{(S) t}(\mathbf{u}, w) . G_{\text {ext } \alpha}^{(S) t}(\mathbf{u}, w)$ then writes

$$
G_{\text {ext } \alpha}^{(S) t}(\mathbf{u}, w)=P_{s c \nu \alpha \alpha}^{(S) t}(\mathbf{u}, w)+P_{s c \nu \alpha \gamma}^{(S) t}(\mathbf{u}, w) .
$$

As the radiative properties associated with the interfaces of an effective phase of a STT or ST2 medium are statistically independent of those of the real semitransparent phase, the variation of the interfacial external scattering cumulative probability between $s^{\prime}$ and $s^{\prime}+\mathrm{d} s^{\prime}$ writes

$$
\mathrm{d} P_{s c \nu \alpha \gamma}^{(S)}(\mathbf{u}, w)=\exp \left(-\beta_{m_{\alpha} \nu} w\right) \frac{\partial}{\partial w} P_{s c \nu \alpha \gamma}^{(S) t}(\mathbf{u}, w) \mathrm{d} s^{\prime} .
$$

The variation of the interfacial internal scattering cumulative probability of an OST, STT or ST2 medium is obtained by replacing $\gamma$ with $\alpha$ in Eq.8. Finally, in the OST case, the variation of the interfacial absorption probability between $s^{\prime}$ and $s^{\prime}+\mathrm{d} s^{\prime}$ is deduced from Eq.8, by replacing $P_{s c \nu \alpha \gamma}^{(S) t}(\mathbf{u}, w)$ with $P_{a \nu \alpha}^{(S) t}(\mathbf{u}, w)$.

Similarly, the variation of the scattering cumulative probability associated with the real semi-transparent phase between $s^{\prime}$ and $s^{\prime}+\mathrm{d} s^{\prime}$ becomes

$$
\mathrm{d} P_{s c \nu m_{\alpha}}^{(S)}(\mathbf{u}, w)=\left[1-G_{e x t \alpha}^{(S) t}(\mathbf{u}, w)\right] \exp \left(-\beta_{m_{\alpha} \nu} w\right) \sigma_{m_{\alpha} \nu} \mathrm{d} s^{\prime} .
$$

There is here no correlation between transmission from $s$ to $s^{\prime}$ and extinction by scattering within $\left[s^{\prime}, s^{\prime}+\mathrm{d} s^{\prime}\right]$, unlike the case of Eq.8. The variations of the absorbing cumulative probability and of the extinction cumulative distribution function associated with the real semi-transparent phase between $s^{\prime}$ and $s^{\prime}+\mathrm{d} s^{\prime}$ are also obtained by replacing in Eq.9 $\sigma_{m_{\alpha} \nu}$ with $\kappa_{m_{\alpha} \nu}$ and $\beta_{m_{\alpha} \nu}$, respectively.

An effective phase is also characterised by a refractive index $n_{\nu \alpha}$ and scattering phase functions $p_{\nu \alpha \alpha}^{(S)}\left(\mathbf{u}_{1}, \mathbf{u}\right)$ and possibly $p_{\nu \alpha \gamma}^{(S)}\left(\mathbf{u}_{1}, \mathbf{u}\right)$. These phase functions a priori depend on the incidence and scattering directions, $\mathbf{u}_{1}$ and $\mathbf{u}$ respectively. A phase function $p_{\nu \alpha}\left(\mu_{s c}\right)$ associated with a real isotropic semi-transparent medium $m_{\alpha}$ only 
depends on the scattering angle cosine $\mu_{s c}$.

\subsubsection{Links between $G_{\text {ext } \alpha}^{S t}$ and $G_{\text {ext } \alpha}^{t}$}

For an effective phase associated with a real phase with diffuse transmission and reflection laws, the extinction cumulative distribution functions $G_{\text {ext } \alpha}^{S t}$ and $G_{\text {ext } \alpha}^{t}$ are linked, as shown in Appendix A, by

$$
\begin{aligned}
w<w_{\min }: G_{\text {ext } \alpha}^{S t}(\mathbf{u}, w) & =0 \\
w>w_{\min }: G_{\text {ext } \alpha}^{S t}(\mathbf{u}, w) & =1-\frac{\partial}{\partial w} G_{e x t \alpha}^{t}(\mathbf{u}, w) /\left(\frac{\partial}{\partial w} G_{e x t \alpha}^{t}(\mathbf{u}, w)\right)_{w_{\min }}
\end{aligned}
$$

where $w_{\text {min }}$ is the smallest distance between two interfacial points. These original equations generalise the results of Ref.[38] to media such that the solid phase is not continuous. Only $G_{\text {ext } \alpha}^{t}(\mathbf{u}, w)$ is practically determined by a numerical Monte Carlo method, as it is much easier to shoot rays within the real phase from random points that belong to a volume than from random interfacial points. $G_{\text {ext } \alpha}^{S t}(\mathbf{u}, w)$ is then deduced from $G_{\text {ext } \alpha}^{t}(\mathbf{u}, w)$ by using Eqs.10.

The internal scattering and external scattering (or absorbing) cumulative probabilities are then given by

$P_{s c \nu \alpha \alpha}^{S t}(\mathbf{u}, w)=\omega_{\nu \alpha \alpha} G_{e x t \alpha}^{S t}(\mathbf{u}, w), P_{s c \nu \alpha \gamma}^{S t}(\mathbf{u}, w)$ or $P_{a \nu \alpha}^{S t}(\mathbf{u}, w)=\left(1-\omega_{\nu \alpha \alpha}\right) G_{\text {ext } \alpha}^{S t}(\mathbf{u}, w)$

where $\omega_{\nu \alpha \alpha}$ is the interfacial albedo equal to $\rho_{\nu \alpha \alpha}$ the interfacial diffuse reflectivity.

\subsubsection{Case of a Beerian effective phase}

If an effective phase is Beerian, it appears from Eqs.10 that $G_{\text {ext } \alpha}^{S t}$ and $G_{\text {ext } \alpha}^{t}$ are identical and write

$$
G_{\text {ext } \alpha}^{S t}(\mathbf{u}, w)=G_{\text {ext } \alpha}^{t}(\mathbf{u}, w)=1-\exp \left[-\beta_{\nu \alpha}(\mathbf{u}) w\right]
$$

where $\beta_{\alpha}(\mathbf{u})$ is the extinction coefficient of the Beerian effective phase $\alpha$. The scattered or absorbed intensities between $s^{\prime}$ and $s^{\prime}+\mathrm{d} s^{\prime}$ are proportional to the initial intensity $I_{\nu \alpha}\left(\mathbf{u}, s^{\prime}\right)$ and to global scattering or absorption coefficients, independently 
of the nature of the radiation source and are not correlated with the transmission from $s$ to $s^{\prime}$. For instance, Equation 8 becomes

$$
\mathrm{d} P_{s c \nu \alpha \gamma}(\mathbf{u}, w)=\exp \left(-\left[\beta_{m_{\alpha} \nu}+\beta_{\alpha}(\mathbf{u})\right] w\right) \sigma_{\nu \alpha \gamma}(\mathbf{u}) \mathrm{d} s^{\prime}
$$

In most of the papers dedicated to the characterisation of OT and OST media, $G_{\text {ext } \alpha}^{t}$ has only been determined instead of $G_{\text {ext } \alpha}^{S t}$, without using Eq10, without an explicit justification[21, 22, 25-27, 29]. This approach is valid insofar the effective phase is at least approximately Beerian[21, 22, 26, 27], even if in some case this assumption is poor[29]. It is also valid even for a strongly non-Beerian effective phase, when the results are only used in the validity conditions of a radiative Fourier law[25], i.e. at a limit such that the phase becomes Beerian.

\subsection{Effective phase with diffuse transmission and reflection laws}

Reflection and transmission are commonly assumed to be diffuse, due to the frequent lack of accurate data but also to the simplicity of the associated modelling. The expressions of an interfacial scattering source term and of the associated scattering phase function are detailed under this assumption in Sec.3.2.1 and that of a correlated interfacial emission source term in Sec.3.2.2, by taking into account the discussions of Sec.2.3.2 and 2.2 respectively.

\subsubsection{Interfacial scattering source terms}

As discussed in Sec.2.3.2 the internal and external scattering phase functions $p_{\nu \alpha \alpha}\left(\mathbf{u}_{1}, \mathbf{u}\right)$ and $p_{\nu \alpha \gamma}\left(\mathbf{u}_{1}, \mathbf{u}\right)$ of an effective phase that is associated with a real phase characterised by diffuse transmission and reflection laws are unique and independent of the history of the incident radiation. Indeed the associated scattered intensities only depend on the global intensity in the incidence direction $\mathbf{u}_{1}$ and on the diffuse bidirectional reflectivity and transmissivity within the real medium[39, 40]. The

phase functions are, in practice, determined in parallel with $G_{\text {ext } \alpha}^{t}(\mathbf{u}, w)$ in a Monte Carlo method based on rays that are shot from random volume points, by determining for all rays the final directions from the transmission and reflection laws [26, 27]. In the case of an STT or ST2 medium, the external scattering phase function 
$p_{\nu \alpha \gamma}\left(\mathbf{u}_{1}, \mathbf{u}\right)$ is the probability per units of incidence and scattering solid angles, for rays incident in the solid angle $\mathrm{d} \Omega_{1 \alpha}\left(\mathbf{u}_{1}\right)$ to be externally scattered in $\mathrm{d} \Omega_{\gamma}(\mathbf{u})$, i.e.

$$
\begin{aligned}
& \frac{p_{\nu \alpha \gamma}\left(\mathbf{u}_{\mathbf{1}}, \mathbf{u}\right)}{4 \pi} \\
& =\frac{\int_{V / \mathbf{u} \cdot \mathbf{n}_{\alpha} \leq 0} \tau_{\nu \alpha \gamma}^{\prime \prime}\left[\mathbf{u}_{\mathbf{1}}, \mathbf{u}, \mathbf{n}_{\alpha}\left(\mathbf{r}, \mathbf{u}_{1}\right)\right]\left[-\mathbf{u}_{\mathbf{1}} \cdot \mathbf{n}_{\alpha}\left(\mathbf{r}, \mathbf{u}_{1}\right)\right] \mathrm{d} \mathbf{r}}{\int_{0}^{4 \pi}\left(\int_{V / \mathbf{u}^{\prime} \cdot \mathbf{n}_{\alpha} \leq 0} \tau_{\nu \alpha \gamma}^{\prime \prime}\left[\mathbf{u}_{\mathbf{1}}, \mathbf{u}^{\prime} \cdot \mathbf{n}_{\alpha}\left(\mathbf{r}, \mathbf{u}_{1}\right)\right]\left[-\mathbf{u}_{\mathbf{1}} \cdot \mathbf{n}_{\alpha}\left(\mathbf{r}, \mathbf{u}_{1}\right)\right] \mathrm{d} \mathbf{r}\right) \mathrm{d} \Omega_{\gamma}^{\prime}\left(\mathbf{u}^{\prime}\right)} .
\end{aligned}
$$

The incidence and scattering directions, $\mathbf{u}_{1}$ and $\mathbf{u}$ respectively, are defined in the fixed frame of the porous medium. The source points $M(\mathbf{r})$ belong to a given volume $V$ of the real phase $\alpha$. The bidirectional transmissivity $\tau_{\nu \alpha \gamma}^{\prime \prime}\left[\mathbf{u}_{\mathbf{1}}, \mathbf{u}, \mathbf{n}_{\alpha}\left(\mathbf{r}, \mathbf{u}_{1}\right)\right]$ is defined in the coordinates of the fixed frame; $\mathbf{n}_{\alpha}\left(\mathbf{r}, \mathbf{u}_{1}\right)$ is the normal unit vector, oriented towards $\alpha$, at the interfacial impact point that is associated with a ray issued from $M(\mathbf{r})$ in the incidence direction $\mathbf{u}_{1}$. The condition $\mathbf{u} . \mathbf{n}_{\alpha} \leq 0$ means that the scattered direction belongs to $\gamma$.

In the case of an OT, OST, STT or ST2 medium, the similar expression of $p_{\nu \alpha \alpha}\left(\mathbf{u}_{1}, \mathbf{u}\right)$ is given for instance in Ref.[26].

Consider now, within a non-Beerian effective phase of an OT, OST, STT or ST2 medium, the interfacial scattering source term $S_{s c \nu \alpha \alpha}^{S t}(\mathbf{u}, s)$ of an interval $[s, s+\mathrm{d} s]$ of axis $\mathbf{u}$, around a point $M(s)$. As discussed in Sec.2.3.2, the fraction of the incident intensity $I_{\nu \alpha}\left(s_{1}^{\prime}, \mathbf{u}_{1}\right)$ at the same point $M\left(s_{1}^{\prime}\right)$, of abscissa $s_{1}^{\prime}$ in the direction $\mathbf{u}_{1}$, that is scattered in the direction $\mathbf{u}$ is not correlated with $I_{\nu \alpha}\left(s_{1}^{\prime}, \mathbf{u}_{1}\right)$. It is proportional to the globally scattered intensity $\omega_{\nu \alpha \alpha} I_{\nu \alpha}\left(s_{1}^{\prime}, \mathbf{u}_{1}\right)$ and to the scattering phase function $p_{\nu \alpha \alpha}\left(\mathbf{u}_{1}, \mathbf{u}\right)$, which is determined by an equation similar to Eq.14. The interfacial scattering source term then writes

$$
S_{s c \nu \alpha \alpha}^{S t}(\mathbf{u}, s)=\frac{\omega_{\nu \alpha \alpha}}{4 \pi} \int_{4 \pi} \frac{\partial}{\partial s_{1}^{\prime}\left(\mathbf{u}_{1}\right)} I_{\nu \alpha}\left(\mathbf{u}_{1}, s_{1}^{\prime}\right) p_{\nu \alpha \alpha}\left(\mathbf{u}_{1}, \mathbf{u}\right) \mathrm{d} \Omega_{1}\left(\mathbf{u}_{1}\right)
$$

As also discussed in Sec.2.3.2, $S_{s c \nu \alpha \alpha}^{S t}$ is not correlated with the following transmissivity $1-G_{\text {ext } \alpha}^{S t}\left(\mathbf{u}, s^{\prime}-s\right)$ from $s$ to $s^{\prime}$ in the direction $\mathbf{u}$. In the case of a STT or ST2 medium, $S_{s c \nu \alpha \gamma}^{S t}(\mathbf{u}, s)$ is obtained by an equation similar to Eq.15, by replacing 
$\omega_{\nu \alpha \alpha}$ with $\omega_{\nu \alpha \gamma}$ and $p_{\nu \alpha \alpha}$ with $p_{\nu \alpha \gamma}$.

3.2.2. Interfacial emission source terms (OT and OST cases)

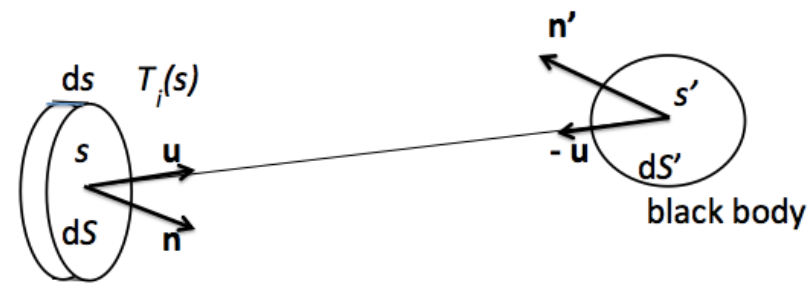

Figure 3: Reciprocal emission and absorption events

For an OT medium, the key problem is to express $\mathrm{d} \mathcal{P}_{\mathrm{d} s s^{\prime} \nu}^{e i}$, power emitted in the elementary solid angle $\mathrm{d} \Omega(\mathbf{u})$ by the interfaces of a volume element $\mathrm{d} V$, equal to $\mathrm{d} S \mathrm{~d} s$, of temperature field $T_{i}(s)$ and incident at $s^{\prime}$ after transmission through the effective phase from $s$ to $s^{\prime}$, as shown in Fig.3. The normal unit vector of $\mathrm{d} S$ is $\mathbf{n}$. This power is also equal to $\mathrm{d} \mathcal{P}_{\mathrm{d} s s^{\prime} \nu}^{e}$, emitted by the interfaces of $\mathrm{d} V$ in $\mathrm{d} \Omega(\mathbf{u})$ and absorbed at the abscissa $s^{\prime}$ by an interfacial element that is assumed to be black, $\mathrm{d} S^{\prime}$ intercepted by $\mathrm{d} \Omega(\mathbf{u})$, of normal unit vector $\mathbf{n}^{\prime}$ and of arbitrary temperature $T_{b}$. The reciprocity theorem states that

$$
w=\left|s^{\prime}-s\right|, \quad \mathrm{d} \mathcal{P}_{\mathrm{d} s s^{\prime} \nu}^{e a}(\mathbf{u}, w)=\mathrm{d} \mathcal{P}_{s^{\prime} \mathrm{d} s \nu}^{e a}(-\mathbf{u}, w) \frac{I_{\nu}^{\circ}\left(T_{i}\right)}{I_{\nu}^{\circ}\left(T_{b}\right)}
$$

It is then sufficient to express $\mathrm{d} \mathcal{P}_{s^{\prime}}^{e} \mathrm{~d} s \nu$, power emitted by the black interfacial element $\mathrm{d} S^{\prime}$ in the reciprocal elementary solid angle $\mathrm{d} \Omega^{\prime}(-\mathbf{u})$ and absorbed by $\mathrm{d} V$, between $s$ and $s+\mathrm{d} s$. By using Eq.16 and the Clausius theorem (invariance of $n_{\nu}^{2} \mathbf{u} . \mathbf{n} \mathrm{d} S \mathrm{~d} \Omega$ ), $\mathrm{d} \mathcal{P}_{\mathrm{d} s s^{\prime} \nu}^{e ~} i$, writes, as $\partial P_{a \nu}^{S t}(-\mathbf{u}, w) / \partial w=\partial P_{a \nu}^{S t}(\mathbf{u}, w) / \partial w$

$$
\mathrm{d} \mathcal{P}_{\mathrm{d} s s^{\prime} \nu}^{e i}(\mathbf{u}, w)=\mathrm{d} \mathcal{P}_{\mathrm{d} s s^{\prime} \nu}^{e a}(\mathbf{u}, w)=\Pi n_{\nu}^{2} I_{\nu}^{\circ}\left(T_{i}\right) \mathrm{d} \nu \mathrm{d} V \mathrm{~d} \Omega \frac{\partial}{\partial w} P_{a \nu}^{S t}(\mathbf{u}, w)
$$

This result is valid for any type of reflection law. It appears in Eq.17 that emission between $s$ and $s+\mathrm{d} s$, as absorption, is strongly correlated with transmission from $s$ to $s^{\prime}$ : It is the signature of a non-Beerian effective phase, as discussed in Sec.3.2.2. 
In the common case of a diffuse reflection law, Equation 17 simply becomes

$$
\mathrm{d} \mathcal{P}_{\mathrm{d} s s^{\prime} \nu}^{e i}(\mathbf{u}, w)=\Pi n_{\nu}^{2} I_{\nu}^{\circ}\left(T_{i}\right) \mathrm{d} \nu \mathrm{d} V \mathrm{~d} \Omega\left(1-\omega_{\nu}\right) \frac{\partial}{\partial w} G_{e x t}^{S t}(\mathbf{u}, w)
$$

and, moreover if the effective phase is Beerian, the following classical result is found again

$$
\left(1-\omega_{\nu}\right) \frac{\partial}{\partial w} G_{e x t}^{S t}(\mathbf{u}, w)=\left(1-\omega_{\nu}\right) \beta_{\nu}\left[1-G_{e x t}^{S t}(\mathbf{u}, w)\right]=\kappa_{\nu}\left[1-G_{e x t}^{S t}(\mathbf{u}, w)\right]
$$

As extinction by the real phase of an OST medium is not correlated with interfacial emission, extinction, absorption or scattering, the additional extinction factor $\exp \left[-\beta_{\nu m} w\right]$ is simply introduced in Eqs. 17 and 18.

\subsection{Non-Beerian effective phase with non-diffuse interfaces}

If transmission and reflection are not diffuse, all the transmission and scattering phenomena are correlated within a non-Beerian effective phase, from an emission point up to any considered point, as discussed in Sec.2.3.3. Moreover, in OT or OST case, interfacial emission is also correlated with all these following phenomena. The case of a STT medium has been pragmatically and numerically developed by Dauvois et al.[31]. The present Section deals with the complete physical formalism for an OST medium.

Consider first the case of emission by the real semi-transparent phase of an OST medium in an elementary solid angle $\mathrm{d} \Omega_{0}$ around the direction $\mathbf{u}_{0}$. A set of specific radiative statistical functions are associated with any elementary path between the initial emission point and the first scattering point and between all the successive scattering points. $G_{e x t \alpha 0}\left(\mathbf{u}_{0}, w_{0}\right), P_{s c \alpha 0}\left(\mathbf{u}_{0}, w_{0}\right)$ and $p_{\nu \alpha \alpha 0}^{H}\left(\mathbf{u}_{0}, \mathbf{u}_{1}\right)$ are the specific extinction cumulative distribution function, scattering cumulative probability and phase function associated with the first path in the direction $\mathbf{u}_{0}$ between the emission point $M_{0}\left(s_{0}\right)$ and the impact point $M_{0}^{\prime}\left(s_{0}^{\prime}\right) ; w_{0}$ is equal to $s_{0}^{\prime}-s_{0}$. Here and in the following, a quantity with a superscript ${ }^{H}$ is correlated with the history of the incident radiation.

The point $M_{0}^{\prime}\left(s_{0}^{\prime}\right)$ becomes a scattering source point that is now called $M_{1}\left(s_{1}\right)$ for 
a scattering direction $\mathbf{u}_{1}$, as $s_{1}$ is its abscissa along the axis $\mathbf{u}_{1} . G^{S H}{ }_{\text {ext } \alpha 1}\left(\mathbf{u}_{1}, w_{1}\right)$, $P_{s c \alpha 1}^{S H}\left(\mathbf{u}_{1}, w_{1}\right)$ and $p_{\nu \alpha \alpha 1}^{S H}\left(\mathbf{u}_{1}, \mathbf{u}_{2}\right)$ are the radiative statistical functions associated with the second path in the direction $\mathbf{u}_{1}$ from the scattering point $M_{1}\left(s_{1}\right)$ to the impact point $M_{1}^{\prime}\left(s_{1}^{\prime}\right) ; w_{1}$ is equal to $s_{1}^{\prime}-s_{1}$. The point $M_{1}^{\prime}\left(s_{1}^{\prime}\right)$ becomes a scattering source point that is now called $M_{2}\left(s_{1}\right)$ for a scattering direction $\mathbf{u}_{2}$, as $s_{2}$ is its abscissa along the axis $\mathbf{u}_{2}$, etc.

The specific extinction cumulative distribution functions $G_{\text {ext } \alpha N}^{S t H}\left(\mathbf{u}_{N}, w_{N}\right)$ associated with a virtual transparent phase (see Sec.3.1.1) writes, by setting: $w_{N}=s_{N}^{\prime}-s_{N}$,

$$
\begin{aligned}
& G_{\text {ext } \alpha N}^{S t H}\left(\mathbf{u}_{N}, w_{N}\right)= \\
& \frac{1}{\sum_{N}^{H}} \frac{1}{\delta \Omega_{\alpha N}\left(\mathbf{u}_{N}\right)} \int_{s_{N}}^{s_{N}^{\prime}} \int_{\delta \Omega_{\alpha N}\left(\mathbf{u}_{N}\right) / \mathbf{u}^{\prime} \cdot \mathbf{n}_{\alpha}>0} \int_{\Sigma_{N}^{H} / V} \delta\left[s^{\prime \prime}-s_{e x t}\left(\mathbf{r}, \mathbf{u}^{\prime}\right)\right] \mathrm{d} \mathbf{r} \mathrm{d} \Omega_{\alpha N}\left(\mathbf{u}^{\prime}\right) \mathrm{d} s^{\prime \prime} .
\end{aligned}
$$

In Eq.20, the summations are carried out:

i) Over all the source points $M(\mathbf{r})$ of the part $\Sigma_{N}^{H}$ of the interfacial surface that belongs to the physical volume $V$ and is impacted by the rays issued from the elementary solid angle $\mathrm{d} \Omega_{\alpha}\left(\mathbf{u}_{N-1}\right)$;

ii) Over all the directions $\mathbf{u}^{\prime}$ of the elementary solid angle $\delta \Omega_{\alpha}\left(\mathbf{u}_{N}\right)$, around the direction $\mathbf{u}_{N}$ of the fixed frame, such that $\mathbf{u}^{\prime}$ belongs to the phase, i.e.: $\mathbf{u}^{\prime} \cdot \mathbf{n}_{\boldsymbol{\alpha}}>0$, where $\mathbf{n}_{\alpha}\left(\mathbf{r}, \mathbf{u}^{\prime}\right)$ is the normal unit vector at an impact point that is oriented towards $\alpha$

iii) Over all the points $s$ " of the axis of direction $\mathbf{u}^{\prime}$, from $M_{N}\left(s_{N}\right)$ to $M_{N}^{\prime}\left(s_{N}^{\prime}\right)$; $s_{\text {ext }}\left(\mathbf{r}, \mathbf{u}^{\prime}\right)$ is the abscissa of the interfacial impact point.

$P_{\text {sc } \alpha \alpha N}^{S t H}\left(\mathbf{u}_{N}, w_{N}\right)$ is obtained in parallel by multiplying the weight $\delta\left[s^{\prime \prime}-s_{\text {ext }}\left(\mathbf{r}, \mathbf{u}^{\prime}\right)\right]$ by the hemispherical reflectivity $[39,40] \rho_{\nu \alpha \alpha}^{\prime h}\left[\mathbf{u}^{\prime}, \mathbf{n}_{\alpha}\left(\mathbf{r}, \mathbf{u}^{\prime}\right)\right]$ in Eq.20. $G_{\text {ext } \alpha N}^{S H}\left(\mathbf{u}_{N}, w_{N}\right)$ is then deduced from $G_{\text {ext } \alpha N}^{S t H}\left(\mathbf{u}_{N}, w_{N}\right)$ by using Eq.5 and $P_{s c \alpha \alpha N}^{S H}\left(\mathbf{u}_{N}, w_{N}\right)$ by using a similar equation.

The specific phase function $p_{\nu \alpha \alpha N}^{S H}\left(\mathbf{u}_{N}, \mathbf{u}_{N+1}\right)$, probability per units of incidence and scattering solid angles for rays that are incident in the solid angle $\mathrm{d} \Omega_{N}\left(\mathbf{u}_{N}\right)$ to 
be internally scattered in $\mathrm{d} \Omega_{N+1}\left(\mathbf{u}_{\mathbf{N}+\mathbf{1}}\right)$, writes for an OST medium

$$
\frac{p_{\nu \alpha \alpha N}^{S H}\left(\mathbf{u}_{N}, \mathbf{u}_{N+1}\right)}{4 \pi}=\frac{\int_{\delta \Omega_{\alpha N}\left(\mathbf{u}_{N}\right) / \mathbf{u}^{\prime} \cdot \mathbf{n}_{\alpha}>0} \int_{\Sigma_{N}^{H} / \mathbf{u}_{\mathbf{N}+\mathbf{1}} \cdot \mathbf{n}_{\alpha} \geq 0} X_{N+1}\left(\mathbf{r}, \mathbf{u}^{\prime}\right) \mathrm{d} \Omega_{\alpha N}\left(\mathbf{u}^{\prime}\right) \mathrm{d} \mathbf{r}}{\int_{0}^{4 \pi}\left(\int_{\delta \Omega_{\alpha N}\left(\mathbf{u}_{N}\right) / \mathbf{u}^{\prime} \cdot \mathbf{n}_{\boldsymbol{\alpha}}>0} \int_{\Sigma_{N}^{H} / \mathbf{u}_{\mathbf{N}+\mathbf{1}}^{\prime} \cdot \mathbf{n}_{\alpha} \geq 0} X_{N+1}\left(\mathbf{r}, \mathbf{u}^{\prime}\right) \mathrm{d} \Omega_{\alpha N}\left(\mathbf{u}^{\prime}\right) \mathrm{d} \mathbf{r}\right) \mathrm{d} \Omega_{N+1}^{\prime}},
$$

with

$$
X_{N+1}\left(\mathbf{r}, \mathbf{u}^{\prime}\right)=\exp \left(-\beta_{\nu m_{\alpha}}\left[s_{e x t}\left(\mathbf{r}, \mathbf{u}^{\prime}\right)-s_{N}(\mathbf{r})\right]\right) \rho_{\nu \alpha \alpha}^{\prime \prime}\left[\mathbf{u}^{\prime}, \mathbf{u}_{N+1}, \mathbf{n}_{\alpha}\left(\mathbf{r}, \mathbf{u}^{\prime}\right)\right]\left[-\mathbf{u}^{\prime} . \mathbf{n}_{\alpha}\left(\mathbf{r}, \mathbf{u}^{\prime}\right)\right]
$$

The summations are carried out in Eq.21, both in the numerator and the denominator, over the source points belonging to $\Sigma_{N}^{H}$ and also over the elementary solid angle $\delta \Omega_{\alpha N}\left(\mathbf{u}_{N}\right)$ for the sake of compatibility with the determination of $G_{\text {ext } \alpha N}^{S t H}\left(\mathbf{u}_{N}, w_{N}\right)$. Moreover the summations are carried out in the denominator over all scattering directions of the phase $\alpha$. The exponential factor in Eq.22 is associated with extinction by the real semi-transparent phase of the OST medium of extinction coefficient $\beta_{\nu m_{\alpha}}$.

Consider now the case of initial interfacial emission at the point $M\left(s_{0}\right)$ within a non-Beerian effective phase of an OST medium. This interfacial emission is correlated with the transmissivity from $M\left(s_{0}\right)$ to the first extiction point $M_{0}^{\prime}\left(s_{0}^{\prime}\right)$ (see Eq. 17) and with a possible scattering at this point, etc. The power emitted by an elementary volume $\mathrm{d} V_{0}$ around $M_{0}\left(s_{0}\right)$ in an elementary solid angle $\mathrm{d} \Omega_{0}$ around the direction $\mathbf{u}_{0}$, transmitted up to $M_{0}^{\prime}\left(s_{0}^{\prime}\right)$ and scattered at this point writes, by generalising Eqs.17 and 20 ,

$$
\begin{aligned}
& \mathrm{d} \mathcal{P}_{\mathrm{d} V_{0} s_{0}^{\prime} \nu}^{e s t}\left(\mathbf{u}_{0}, w_{0}\right)=\Pi_{\alpha} n_{\nu}^{2} I_{\nu}^{\circ}\left(T_{i}\right) \mathrm{d} \nu \mathrm{d} V \mathrm{~d} \Omega \\
& \mathrm{x} \quad \frac{1}{\Sigma_{N}^{H}} \frac{1}{\delta \Omega_{\alpha 0}\left(\mathbf{u}_{0}\right)} \frac{\partial}{\partial w_{0}} \int_{s_{0}}^{s_{0}^{\prime}} \int_{\delta \Omega_{\alpha}\left(\mathbf{u}_{0}\right) / \mathbf{u}^{\prime} . \mathbf{n}_{\boldsymbol{\alpha}}>0} \int_{\Sigma / V} \exp \left(-\beta_{m_{\alpha} \nu} w_{0}\right) Y\left(\mathbf{r}, \mathbf{u}^{\prime}, s^{\prime \prime}\right) \mathrm{d} \mathbf{r} \mathrm{d} \Omega_{\alpha 0}\left(\mathbf{u}^{\prime}\right) \mathrm{d} s^{\prime \prime}
\end{aligned}
$$

with

$$
Y\left(\mathbf{r}, \mathbf{u}^{\prime}, s^{\prime \prime}\right)=\left(1-\rho_{\nu \alpha \alpha}^{\prime h}\left[\mathbf{u}^{\prime}, \mathbf{n}_{\alpha}\left(\mathbf{r}, \mathbf{u}^{\prime}\right)\right]\right) \rho_{\nu \alpha \alpha}^{\prime h}\left[\mathbf{u}^{\prime}, \mathbf{n}_{\alpha}\left(\mathbf{r}, \mathbf{u}^{\prime}\right)\right] \delta\left[s^{\prime \prime}-s_{e x t}\left(\mathbf{r}, \mathbf{u}^{\prime}\right)\right]
$$


$p_{\nu \alpha \alpha 0}^{S i H}\left(\mathbf{u}_{0}, \mathbf{u}_{1}\right), G_{s c, \alpha 1}^{S i H}\left(\mathbf{u}_{1}, w_{1}\right), P_{s c, \alpha \alpha 1}^{S i H}\left(\mathbf{u}_{1}, w_{1}\right), p_{\nu \alpha \alpha 1}^{S t i H}\left(\mathbf{u}_{1}, \mathbf{u}_{2}\right)$, etc. are then determined as in the previous case of emission by the semi-transparent real phase $m_{\alpha}$ but differ from the previous corresponding quantities.

\subsection{Non-homogeneous effective phase}
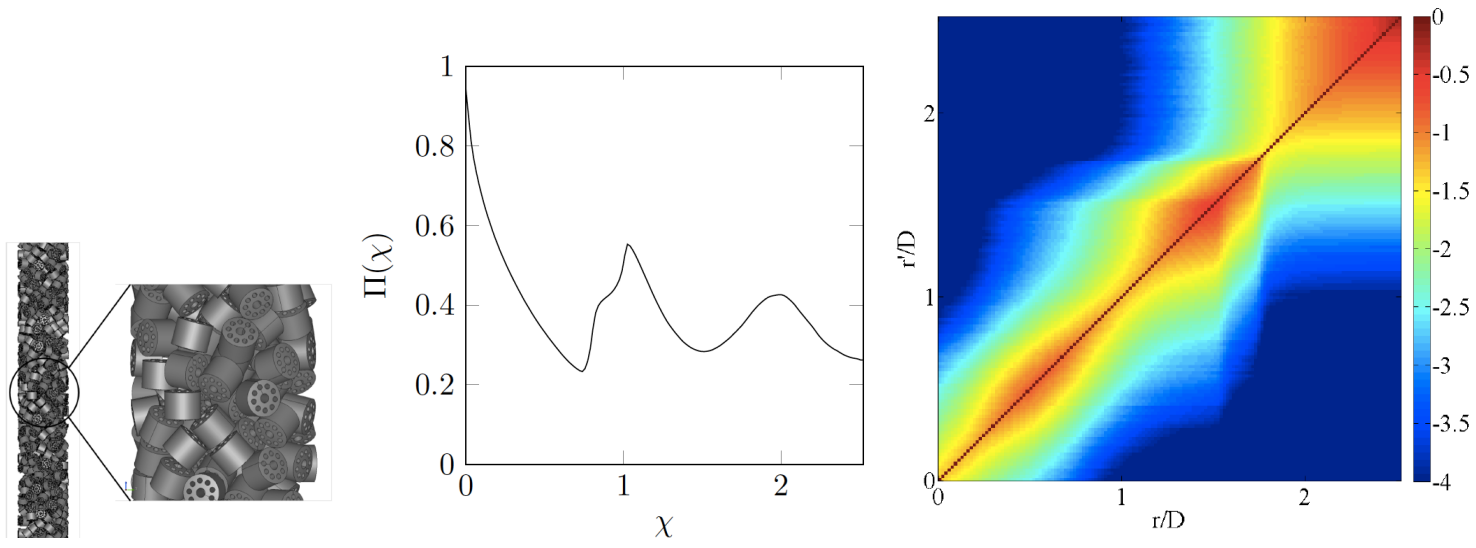

Figure 4: Left: Set of non-overlapping opaque cylindrical pellets of diameter $D$ and height $H=0.8 D$ enclosed in a tube of radius $R_{t}=2.5 D[41-43]$; Middle: Porosity field $\Pi$ vs $\chi=\left(R_{t}-r\right) / D, r$ radial coordinate; Right: $\ln \left(\Pi(r)\left[1-G_{e x t}^{t}\left(r, \mathbf{e}_{r}, r^{\prime}\right)\right]\right)$ in a tube cross section; $\mathbf{e}_{r}$ radial unit vector.

For the sake of clarity, this Section is only devoted to non-homogeneous effective phases $\alpha$ associated with real phases with diffuse transmission and reflection laws. For instance, the presence of a wall often generates a non-homogeneity within a porous medium. It is the case of a set of pellets confined by a wall, as shown in Fig.4[30]. Such media are characterised by strong gradients of the volume fraction $\Pi_{\alpha}(M)$ which is assumed to be continuous. They often present, in cartesian or cylindrical coordinates, one or two dimensions that are much larger than the other one(s) and along which they are statistically homogeneous.

The key quantities are radiative statistical distribution functions that also depend on the position of the scattering or emission source point $M(s)$, such as $G_{\text {ext } \alpha \nu}^{(S)}\left(\mathbf{u}, s, s^{\prime}-s\right)$ or $G_{\text {ext } \alpha \nu}^{(S)}\left(\mathbf{u}, M, M^{\prime}\right)$. A non-homogeneous effective phase is never Beerian, due to this dependence of $G_{\text {ext } \alpha \nu}^{(S)}\left(\mathbf{u}, M, M^{\prime}\right)$ vs $s$. Moreover, within an effective phase of a non-homogeneous medium the classical transmissivity 1 - 
$G_{\text {ext } \alpha \nu}^{(S)}\left(\mathbf{u}, M, M^{\prime}\right)$ is not invariant by time reversal. According to the reciprocity the-

orem, only the generalised transmissivity $\Pi_{\alpha}(M)\left[1-G_{\text {ext } \alpha \nu}^{(S)}\left(\mathbf{u}, M, M^{\prime}\right)\right]$ is invariant by time reversal[30], i.e.

$$
\Pi_{\alpha}(M)\left[1-G_{\text {ext } \alpha}^{(S)}\left(\mathbf{u}, M, M^{\prime}\right)\right]=\Pi_{\alpha}\left(M^{\prime}\right)\left[1-G_{\text {ext } \alpha}^{(S)}\left(-\mathbf{u}, M^{\prime}, M\right)\right] .
$$

All the developments of Sec.3 remain valid if they are based on the generalised transmissivity instead of the classical one and on similar quantities associated with scattering and absorption. This approach has been implemented and applied to radiative transfer for an OT case, a set of opaque spheres confined at the vicinity of plane walls[30], by taking into account interfacial emission correlated with transmission. Figure 4 is an illustration of Eq.25, issued from a parallel study dedicated to an axi-symmetrical system[43].

\section{Generalised Radiative Transfer Equation (diffuse interfacial laws)}

The Generalised Radiative Transfer Equations (GRTEs) that are associated with non-Beerian homogeneous effective phases of OT, OST, ST2 and STT media with diffuse interfacial transmission and reflection laws are presented in this Section. As discussed in Sec.2.4, they are based on the temperature field $T_{i}(\mathbf{r})$ of the interfaces (OT medium), on $T_{i}(\mathbf{r})$ and the temperature field $T_{\alpha}(\mathbf{r})$ of the effective phase $\alpha$ (OST medium), on $T_{\alpha}(\mathbf{r})$ (STT medium) or on $T_{\alpha}(\mathbf{r})$ and $T_{\gamma}(\mathbf{r})$ (ST2 medium). The GRTEs associated with models based on a global temperature field can easily be deduced from these GRTEs.

The more complex case of an effective phase associated with a real phase with nondiffuse reflection or transmission law has been discussed in Sec.3.3.

\subsection{OT medium}

A homogeneous effective phase $\alpha$ of an OT medium with a diffuse interfacial reflection law is characterised by the isotropic albedo $\omega_{\nu \alpha \alpha}$. The emission source term that is correlated with the following transmissivity has been expressed in Sec.3.2.2, 
the scattering source term and the associated phase function in Sec.3.2.1. The associated GRTE then writes by setting: $w=s^{\prime}-s$,

$$
\begin{aligned}
I_{\nu \alpha}\left(\mathbf{u}, \mathbf{s}^{\prime}\right) & =\int_{s_{b}}^{s^{\prime}}\left(1-\omega_{\nu \alpha \alpha}\right) \Pi_{\alpha} n_{\nu \alpha}^{2} I_{\nu}^{\circ}\left[T_{i}(s)\right] \frac{\partial}{\partial w} G_{e x t \alpha}^{S t}(\mathbf{u}, w) \mathrm{d} s \\
& +\int_{s_{b}}^{s^{\prime}} S_{s c \nu \alpha \alpha}^{S t}(\mathbf{u}, s)\left[1-G_{\text {ext } \alpha}^{S t}(\mathbf{u}, w)\right] \mathrm{d} s+I_{\nu \alpha}\left(\mathbf{u}, s_{b}\right)\left[1-G_{\text {ext } \alpha}^{S_{b} t}\left(\mathbf{u}, w_{b}\right)\right] .
\end{aligned}
$$

This equation was first introduced in Ref.[34], but for a model that was limited to the core of the porous medium, and also in Ref.[38] but with a copy-paste mistake. The first term of the right side of Eq.26 is the sum from the porous medium boundary $s_{b}$ to $s^{\prime}$ of the intensities that are emitted by the interfaces between $s$ and $s+\mathrm{d} s$ in the direction $\mathbf{u}$ and transmitted up to the point $s^{\prime}$ (see Eq.18).

The second term of the right side of Eq.26 is the sum from $s_{b}$ to $s^{\prime}$ of the intensities that are incident from all directions of the space at the point $M(s)$, scattered at $s$ in the direction $\mathbf{u}$ and transmitted from $s$ to $s^{\prime}$. As discussed in Sec.3.2.1, the scattering source term, given by Eq.15, is not correlated with the incident intensity distribution, but makes the GRTE extremely implicit, i.e.

$$
S_{s c \nu \alpha \alpha}^{S t}(\mathbf{u}, s)=\frac{\omega_{\nu \alpha \alpha}}{4 \pi} \int_{4 \pi} \frac{\partial}{\partial s_{1}^{\prime}\left(\mathbf{u}_{1}\right)} I_{\nu \alpha}\left(\mathbf{u}_{1}, s_{1}^{\prime}\right) p_{\nu \alpha \alpha}\left(\mathbf{u}_{1}, \mathbf{u}\right) \mathrm{d} \Omega_{1}\left(\mathbf{u}_{1}\right)
$$

where $s_{1}^{\prime}\left(\mathbf{u}_{1}\right)$ is the abscissa in the incidence direction $\mathbf{u}_{1}$ of the point $M$ of abscissa $s$ in the considered direction $\mathbf{u}$.

The last term of the right side of Eq.26 represents the intensity that is transmitted in the direction $\mathbf{u}$ from a boundary point $M_{b}\left(s_{b}\right)$ to $M^{\prime}\left(s^{\prime}\right)$. It is assumed that $I_{\nu \alpha}\left(\mathbf{u}, s_{b}\right)$ is not correlated with $\left[1-G_{\text {ext } \alpha}^{S_{b} t}\left(\mathbf{u}, w_{b}\right)\right]$ (case a wall with a diffuse reflection law, for instance).

Equations 26 and 27 are directly expressed vs cumulative distribution functions, which is the requirement of a Monte Carlo method. The GRTE is then as easily solved as a classical RTE. [30, 31]. 


\subsection{OST medium}

An effective phase $\alpha$ of an OST medium with a diffuse interfacial reflection law is characterised by five source terms, associated with emission and scattering by the interfaces and the real semi-transparent medium $m_{\alpha}$, and also with the radiation issued from the boundaries. As previously, correlations only occur between interfacial

phenomena. The corresponding original GRTE, in which $G_{\text {ext } \alpha}^{S}$ and $G_{\text {ext } \alpha}$ are given by Eq. 5 and the interfacial scattering source term $S_{s c \nu \alpha \alpha}^{S}(\mathbf{u}, s)$ is also given by Eq.15, writes

$$
\begin{aligned}
I_{\nu \alpha}\left(\mathbf{u}, \mathbf{s}^{\prime}\right) & =\int_{s_{b}}^{s^{\prime}}\left(1-\omega_{\nu \alpha \alpha}\right) \Pi_{\alpha} n_{\nu \alpha}^{2} I_{\nu}^{\circ}\left[T_{i}(s)\right] \frac{\partial}{\partial w} G_{e x t \alpha}^{S}(\mathbf{u}, w) \mathrm{d} s \\
& +\int_{s_{b}}^{s^{\prime}} \kappa_{\nu m_{\alpha}} \Pi_{\alpha} n_{\nu \alpha}^{2} I_{\nu}^{\circ}\left[T_{\alpha}(s)\right]\left[1-G_{e x t \alpha}\left(\mathbf{u}, s^{\prime}-s\right)\right] \mathrm{d} s \\
& +\int_{s_{b}}^{s^{\prime}} S_{s c \nu \alpha \alpha}^{S}(\mathbf{u}, s)\left[1-G_{e x t \alpha}^{S}\left(\mathbf{u}, s^{\prime}-s\right)\right] \mathrm{d} s \\
& +\int_{s_{b}}^{s^{\prime}}\left(\frac{\sigma_{\nu m_{\alpha}}}{4 \pi} \int_{0}^{4 \pi} p_{\nu m_{\alpha}}\left(\mathbf{u}_{1} \cdot \mathbf{u}\right) I_{\nu}\left[\mathbf{u}_{1}, s_{1}^{\prime}\left(\mathbf{u}_{1}\right)\right] \mathrm{d} \Omega_{1}\left(\mathbf{u}_{1}\right)\right)\left[1-G_{e x t \alpha}\left(\mathbf{u}, s^{\prime}-s\right)\right] \mathrm{d} s \\
& +I_{\nu \alpha}\left(\mathbf{u}, s_{b}\right)\left[1-G_{\text {ext } \alpha}^{S_{b}}\left(\mathbf{u}, s^{\prime}-s_{b}\right)\right] .
\end{aligned}
$$

\subsection{ST2 and STT media}

The expression of the GRTE of a non-Beerian effective phase $\alpha$ of a ST2 medium with diffuse interfacial transmission and reflection laws coupled with the GRTE of the effective phase $\gamma$ involves five different source terms, i.e. emission and scattering source terms of the real semi-transparent medium $m_{\alpha}$, internal interfacial scattering source term $S_{s c \nu \alpha \alpha}^{S}$ of $\alpha$, external interfacial scattering source term $S_{s c \nu \gamma \alpha}^{S}$ from $\gamma$ to $\alpha$ and intensity transmitted from the porous medium boundaries, which is assumed to be non-correlated with the folowing transmissivity. This GRTE has not been 
implemented to our knowledge.

$$
\begin{aligned}
I_{\nu \alpha}\left(\mathbf{u}, s^{\prime}\right) & =\int_{s_{b}}^{s^{\prime}} S_{\nu m_{\alpha}}(\mathbf{u}, s)\left[1-G_{e x t \alpha}\left(\mathbf{u}, s^{\prime}-s\right)\right] \mathrm{d} s+I_{\nu \alpha}\left(\mathbf{u}, s_{b}\right)\left[1-G_{e x t \alpha}^{S_{b}}\left(\mathbf{u}, s^{\prime}-s_{b}\right)\right] \\
& +\int_{s_{b}}^{s^{\prime}}\left[S_{s c \nu \alpha \alpha}^{S}(\mathbf{u}, s)+S_{s c \nu \gamma \alpha}^{S}(\mathbf{u}, s)\right]\left[1-G_{e x t \alpha}^{S}\left(\mathbf{u}, s^{\prime}-s\right)\right] \mathrm{d} s
\end{aligned}
$$

where the total source term within $m_{\alpha}$ writes

$$
S_{\nu m_{\alpha}}(\mathbf{u}, s)=\Pi_{\alpha} \kappa_{\nu m_{\alpha}} n_{\nu \alpha}^{2} I_{\nu}^{\circ}\left[T_{\alpha}(s)\right]+\frac{\sigma_{\nu m_{\alpha}}}{4 \pi} \int_{0}^{4 \pi} p_{\nu m_{\alpha}}\left(\mathbf{u}_{1} \cdot \mathbf{u}\right) I_{\nu \alpha}\left[\mathbf{u}_{1}, s_{1}^{\prime}\left(\mathbf{u}_{1}\right)\right] \mathrm{d} \Omega_{1 \alpha}\left(\mathbf{u}_{1}\right)
$$

and the external interfacial scattering source term of $\gamma$

$$
S_{s c \nu \gamma \alpha}^{S}(\mathbf{u}, s)=\frac{\omega_{\nu \gamma \alpha}}{4 \pi} \int_{4 \pi} \frac{\partial I_{\nu \gamma}\left(\mathbf{u}_{1}, s_{1}^{\prime}\right)}{\partial s_{1}^{\prime}\left(\mathbf{u}_{1}\right)} p_{\nu \gamma \alpha}\left(\mathbf{u}_{1}, \mathbf{u}\right) \mathrm{d} \Omega_{1 \gamma}\left(\mathbf{u}_{1}\right)
$$

As previously $s_{1}^{\prime}$ is the abscissa in the direction $\mathbf{u}_{1}$ of the point $M$ of abscissa $s$ in the considered direction $\mathbf{u}$. As the two phases are characterised by different refractive indices, the solid angles $\mathrm{d} \Omega_{\gamma}$ and $\mathrm{d} \Omega_{\alpha}$ at a scattering point differ. All the associated transformations are ruled by the Clausius theorem.

The GRTEs associated with the effective phases of a STT medium are simply obtained by setting: $\beta_{m \gamma}=\sigma_{m \gamma}=\kappa_{m \gamma}=0$, as the $\gamma$ phase is transparent.

\subsection{Degeneration into RTE}

In the core of a porous medium and in regions where a non-Beerian effective phase is practically isothermal along an optically thick distance, the GRTE associated with this effective phase degenerates into a classical RTE, as shown in in Sec.4.4.1 in the case of an OT medium. These conditions correspond to a Local Thermal Equilibrium of Radiation (LTER), which is similar to the Local Thermal Equilibrium of Matter (LTEM) of the molecular theory $[44,45]$. The LTER is introduced in Sec.4.4.2 by comparison with the Ideal Thermal Equilibrium (ITE) defined for radiation in Sec.4.4.1. The cases of OT, OST, STT and ST2 media are discussed in Secs.4.4.3 and 4.4.4. 
The main practical interest of this Section is to be the theoretical base of the popular model of the radiative Fourier law developed in Sec.5.

\subsubsection{Ideal Thermal Equilibrium}

Consider the case of the non-Beerian effective phase $\alpha$ of an OT medium which is characterised by an emission term that is correlated with the following transmission of radiation (see Eq.26). This fact was not accounted for in a previous study[34]. At Ideal Thermal Equilibrium (ITE) the effective phase $\alpha$, of volume fraction $\Pi_{\alpha}$ and refractive index $n_{\nu \alpha}$, is characterised by the equilibrium intensity $\Pi_{\alpha} n_{\nu \alpha}^{2} I_{\nu}^{\circ}(T)$ that is independent of the position. The second term of the right side of Eq.26 vanishes at a distance from the boundaries that is optically thick and this equation becomes

$$
\Pi_{\alpha} n_{\nu \alpha}^{2} I_{\nu}^{\circ}(T)=\left(1-\omega_{\nu \alpha \alpha}\right) \Pi_{\alpha} n_{\nu \alpha}^{2} I_{\nu}^{\circ}(T)+S_{s c \nu \alpha \alpha}^{S t \circ} \int_{0}^{\infty}\left[1-G_{e x t \alpha}^{S t}(\mathbf{u}, w)\right] \mathrm{d} w .
$$

In these conditions, the equilibrium scattering source term writes at any point of the effective phase

$$
S_{s c \nu \alpha \alpha}^{S t \circ}(\mathbf{u}, T)=\frac{\omega_{\nu \alpha \alpha} \Pi_{\alpha} n_{\nu \alpha}^{2} I_{\nu}^{\circ}(T)}{\int_{0}^{\infty}\left[1-G_{\text {ext } \alpha}^{S t}(\mathbf{u}, w)\right] \mathrm{d} w},
$$

and the equilibrium emission source term is then deduced from Eqs. 33 and 26

$$
S_{e \nu \alpha \alpha}^{S t \circ}(\mathbf{u}, T)=\frac{\left(1-\omega_{\nu \alpha \alpha}\right) \Pi_{\alpha} n_{\nu \alpha}^{2} I_{\nu}^{\circ}(T)}{\int_{0}^{\infty}\left[1-G_{e x t \alpha}^{S t}(\mathbf{u}, w)\right] \mathrm{d} w}
$$

In ITE conditions the terms of extinction by scattering and by absorption are equal to the opposites of the scattering and emission source terms, respectively. By taking into account Eqs. 33 and 34, they write $-\Sigma_{\nu \alpha}(\mathbf{u}) \Pi_{\alpha} n_{\nu \alpha}^{2} I_{\nu}^{\circ}(T)$ and $-K_{\nu \alpha}(\mathbf{u}) \Pi_{\alpha} n_{\nu \alpha}^{2} I_{\nu}^{\circ}(T)$, by definition of the generalised scattering and absorption coefficients at equilibrium

$$
\Sigma_{\nu \alpha}(\mathbf{u})=\frac{\omega_{\nu \alpha \alpha}}{\int_{0}^{\infty}\left[1-G_{e x t \alpha}^{S t}(\mathbf{u}, w)\right] \mathrm{d} w} \quad \text { and } \quad K_{\nu \alpha}(\mathbf{u})=\frac{1-\omega_{\nu \alpha \alpha}}{\int_{0}^{\infty}\left[1-G_{e x t \alpha}^{S t}(\mathbf{u}, w)\right] \mathrm{d} w}
$$


The generalised extinction coefficient at equilibrium that then writes

$$
B_{\alpha}(\mathbf{u})=\frac{1}{\int_{0}^{\infty}\left[1-G_{e x t \alpha}^{S t}(\mathbf{u}, w)\right] \mathrm{d} w}
$$

is the inverse of a quantity averaged along an optically thick region, the area under the curve associated with the transmissivity $1-G_{e x t \alpha}^{S t}(\mathbf{u}, w)$. For a Beerian effective phase, $1-G_{\text {ext } \alpha}^{S t}(\mathbf{u}, w)$ is equal to $1-\exp \left[-\beta_{\alpha}(\mathbf{u}) w\right]$ and $B_{\alpha}(\mathbf{u})$ is equal to $\beta_{\alpha}(\mathbf{u})$.

Similar results are easily obtained in OST, STT and ST2 cases. In conclusion, the GRTE associated with a non-Beerian homogeneous effective phase degenerates, in ITE conditions, into a classical RTE that is characterised by the previously defined coefficients at equilibrium.

A non-homogeneous effective phase is characterised by an extinction cumulative distribution function $G_{e x t \nu \alpha}^{(S)}(\mathbf{u}, s, w)$ that depends on the position $s$. In ITE conditions, the total source term is then non-uniform and generalised extinction, absorption and scattering coefficients at equilibrium can not be defined. Consequently, a RTE can never be defined in this case.

\subsubsection{Local Thermal Equilibrium of Radiation}

It is now assumed, as in the Chapman-Enskog theory applied to a molecular assembly[44, 45], that the intensity field $I_{\nu \alpha}(\mathbf{u}, \mathbf{r})$ within an effective phase $\alpha$ is practically characterised at a spatial scale that is optically thick by an equilibrium intensity $\Pi_{\alpha} n_{\alpha}^{2} I_{\nu}^{\circ}(T)$ at the temperature $T$, which only differs from the real intensity field $I_{\nu \alpha}(\mathbf{u}, \mathbf{r})$ by a perturbation term that is neglected in this Section. This assumption corresponds to a Local Thermal Equilibrium of Radiation (LTER), similar to the Local Thermal Equilibrium of Matter (LTEM) of the molecular theory. Note that, within a transparent real phase, this LTER condition can be realised even if the fluid of this phase is strongly non-isothermal, but obviously in LTEM conditions. As seen in this example, the scales of LTER and LTEM conditions are extremely different.

When an effective phase is in LTER conditions, it is characterised by physical quantities defined in ITE conditions, as in LTEM conditions. In particular, a classical RTE based on the extinction, absorption and scattering coefficients at equilibrium 
defined in Sec.4.4.1 is then introduced, as shown in Sec.4.4.3 for an OST or OT medium and in Sec.4.4.3 for a ST2 medium.

The strict validity conditions of this model are discussed in Sec.5 in which the discrepancies to equilibrium conditions are studied and which is mainly devoted to the radiative Fourier law and its validity conditions in Sec.5.1.

\subsubsection{OST or OT medium}

Consider first the effective phase of an OST medium. When the temperature fields of the real semi-transparent phase $m_{\alpha}$ and the interfaces differ a predominant radiative transfer occurs between the interfaces and the phase $m_{\alpha}$ at an optically thin distance. A RTE with two emission sources at different temperatures, as done in Ref.[25], is then not justified. In these conditions, the GRTE defined by Eq.28, which presents two emission terms, has to be solved.

On the contrary, if the interfaces and the phase $m_{\alpha}$ are characterised by the same temperature field $T$ and are practically isothermal along an optically thick distance, a global RTE is valid as discussed in the introduction of Sec.4.4.2, i.e.

$$
\begin{aligned}
\frac{\mathrm{d}}{\mathrm{d} s} I_{\nu \alpha}(\mathbf{u}, s)= & \left.-\left[B_{\alpha}(\mathbf{u})+\beta_{\nu m_{\alpha}}\right] I_{\nu \alpha}(\mathbf{u}, s)+\Pi_{\alpha} n_{\nu \alpha}^{2}\left[K_{\nu \alpha}(\mathbf{u})+\kappa_{\nu m_{\alpha}}\right] I_{\nu}^{\circ}\left[T_{(} s\right)\right] \\
& +\frac{\sigma_{\nu m_{\alpha}}}{4 \pi} \int_{0}^{4 \pi} p_{\nu m_{\alpha}}\left(\mathbf{u}_{1} \cdot \mathbf{u}\right) I_{\nu \alpha}\left[\mathbf{u}_{1}, s_{1}^{\prime}\left(\mathbf{u}_{1}\right)\right] \mathrm{d} \Omega_{1}\left(\mathbf{u}_{1}\right) \\
& +\frac{\Sigma_{\nu \alpha \alpha}(\mathbf{u})}{4 \pi} \int_{0}^{4 \pi} p_{\nu \alpha \alpha}\left(-\mathbf{u},-\mathbf{u}_{1}\right) I_{\nu \alpha}\left[\mathbf{u}_{1}, s_{1}^{\prime}\left(\mathbf{u}_{1}\right)\right] \mathrm{d} \Omega_{1}\left(-\mathbf{u}_{1}\right),
\end{aligned}
$$

where $B_{\alpha}(\mathbf{u}), K_{\nu \alpha}(\mathbf{u}), \Sigma_{\nu \alpha}(\mathbf{u})$ and $p_{\nu \alpha \alpha}\left(-\mathbf{u},-\mathbf{u}_{1}\right)$ are the interfacial generalised extinction, absorption and scattering coefficients at equilibrium and the interfacial scattering phase function; $\beta_{\nu m_{\alpha}}, \kappa_{\nu m_{\alpha}}$ and $\sigma_{\nu m_{\alpha}}$ and $p_{\nu m_{\alpha}}$ the extinction, absorption and scattering coefficients and the scattering phase function of the phase $m_{\alpha}$.

In Eq.37, $\Sigma_{\nu \alpha \alpha}\left(\mathbf{u}_{1}\right)$ has been replaced by $\Sigma_{\nu \alpha \alpha}(\mathbf{u})$, due to using the invariance of the flux at equilibrium by time reversal (see Appendix B) and $\Sigma_{\nu \alpha \alpha}(\mathbf{u})$ has been extracted from the directional integration. The resulting expression of the last scattering source term of Eq.37, which is more general than the expression in use in Ref.[25], is proofed in this Appendix. 
The RTE associated with an OT case is obviously deduced by setting: $\beta_{\nu m_{\alpha}}=$ $\kappa_{\nu m_{\alpha}}=\sigma_{\nu m_{\alpha}}=0$.

\subsubsection{ST2 medium}

In the case of a STT medium, if the effective phase corresponding to the transparent real phase is non-Beerian, a RTE can not can not be associated with it. Indeed, the absorption optical thickness of this effective phase is always zero, never optically thick.

In the case of a ST2 medium, as the assumption of optically thick effective phases is never verified due to the predominant transfer between the two phases associated with the external scattering terms, a model that is based on two coupled RTEs is not valid for non-Beerian effective phases when the temperature fields of the two phases differ. The two coupled GRTEs, given by Eqs.29-31, have then to be solved.

In regions where the two phases have the same temperature field and are practically isotermal along an optically thick distance, a porous medium of ST2 type is globally modelled by considering a unique effective semi-transparent phase of uniform intensity $I_{\nu}$ and effective refractive index $n_{\nu}$. The intensities, refractive indices and elementary solid angles associated with the two phases, the effective medium and the void, characterised by a subscript $v$ are linked by

$$
\begin{aligned}
& I_{\nu}=n_{\nu}^{2} I_{\nu v} \quad I_{\nu \alpha}=\Pi_{\alpha} n_{\nu \alpha}^{2} I_{\nu v}, \quad I_{\nu \gamma}=\Pi_{\gamma} n_{\nu \gamma}^{2} I_{\nu v}, \\
& n_{\nu}^{2}=\Pi_{\alpha} n_{\nu \alpha}^{2}+\Pi_{\gamma} n_{\nu \gamma}^{2}, \quad n_{\nu}^{2} \mathrm{~d} \Omega=\mathrm{d} \Omega_{v}=n_{\nu \alpha}^{2} \mathrm{~d} \Omega_{\alpha}=n_{\nu \gamma}^{2} \mathrm{~d} \Omega_{\gamma}, \\
& I_{\nu} \mathrm{d} \Omega=I_{v} \mathrm{~d} \Omega_{v}, \quad I_{\nu \alpha} \mathrm{d} \Omega_{\alpha}=\Pi_{\alpha} I_{\nu} \mathrm{d} \Omega \quad I_{\nu \gamma} \mathrm{d} \Omega_{\gamma}=\Pi_{\gamma} I_{\nu} \mathrm{d} \Omega
\end{aligned}
$$

Indeed, the intensity expressed in void is uniform within the two real phases. A 
unique RTE is obtained from the two coupled Eqs. B.10 of Appendix B, i.e

$$
\begin{aligned}
\frac{\mathrm{d}}{\mathrm{d} s} I_{\nu}(\mathbf{u}, s)= & -B_{\nu}(\mathbf{u}) I_{\nu}(\mathbf{u}, s)+\kappa_{\nu} n_{\nu}^{2} I_{\nu}^{\circ}[T(s)] \\
& +\frac{\sigma_{\nu}}{4 \pi} \int_{0}^{4 \pi} p_{\nu}\left(\mathbf{u}_{1}, \mathbf{u}\right) I_{\nu}\left[\mathbf{u}_{1}, s_{1}^{\prime}\left(\mathbf{u}_{1}\right)\right] \mathrm{d} \Omega\left(\mathbf{u}_{1}\right) \\
& +\frac{\sum_{\nu i}(\mathbf{u})}{4 \pi} \int_{0}^{4 \pi} p_{\nu i}\left(-\mathbf{u},-\mathbf{u}_{1}\right) I_{\nu}\left[\mathbf{u}_{1}, s_{1}^{\prime}\left(\mathbf{u}_{1}\right)\right] \mathrm{d} \Omega\left(-\mathbf{u}_{1}\right)
\end{aligned}
$$

where the global effective extinction and absorption coefficients $B_{\nu}$ and $\kappa_{\nu}$ and the effective scattering coefficients coefficient $\sigma_{\nu}$ and $\Sigma_{\nu i}$ and phase functions $p_{\nu}$ and $p_{\nu i}$ associated with the phases $m_{\alpha}$ and $m_{\gamma}$ and the interfaces are defined by

$$
\begin{aligned}
n_{\nu}^{2} \kappa_{\nu} & =\Pi_{\alpha} n_{\nu \alpha}^{2} \kappa_{\nu m_{\alpha}}+\Pi_{\gamma} n_{\nu \gamma}^{2} \kappa_{\nu m_{\gamma}} \\
n_{\nu}^{2} \sigma_{\nu} & =\Pi_{\alpha} n_{\nu \alpha}^{2} \sigma_{\nu m_{\alpha}}+\Pi_{\gamma} n_{\nu \gamma}^{2} \sigma_{\nu m_{\gamma}} \\
n_{\nu}^{2} \Sigma_{\nu i}(\mathbf{u}) & =\Pi_{\alpha} n_{\nu \alpha}^{2}\left[\Sigma_{\nu \alpha \alpha}(\mathbf{u})+\Sigma_{\nu \alpha \gamma}(\mathbf{u})\right]+\Pi_{\gamma} n_{\nu \gamma}^{2}\left[\Sigma_{\nu \gamma \gamma}(\mathbf{u})+\Sigma_{\nu \gamma \alpha}(\mathbf{u})\right] \\
B_{\nu}(\mathbf{u}) & =\kappa_{\nu}+\sigma_{\nu}+\Sigma_{\nu i}(\mathbf{u}) \\
\sigma_{\nu} p_{\nu}\left(\mathbf{u}_{1} \cdot \mathbf{u}\right) & =\Pi_{\alpha} \sigma_{\nu m_{\alpha}} p_{\nu m_{\alpha}}\left(\mathbf{u}_{1} \cdot \mathbf{u}\right)+\Pi_{\gamma} \sigma_{\nu m_{\gamma}} p_{\nu m_{\gamma}}\left(\mathbf{u}_{1} \cdot \mathbf{u}\right) \\
\Sigma_{\nu i}(\mathbf{u}) p_{\nu i}\left(-\mathbf{u},-\mathbf{u}_{1}\right) & =\Pi_{\alpha} \Sigma_{\nu \alpha \alpha}(\mathbf{u}) p_{\nu \alpha \alpha}\left(-\mathbf{u},-\mathbf{u}_{1}\right) \\
& +\left(\frac{n_{\nu \alpha}^{2}}{n_{\nu \gamma}^{2}}\right) \Pi_{\alpha} \Sigma_{\nu \alpha \gamma}(\mathbf{u}) p_{\nu \alpha \gamma}\left(-\mathbf{u},-\mathbf{u}_{1}\right) \\
& +\Pi_{\gamma} \Sigma_{\nu \gamma \gamma}(\mathbf{u}) p_{\nu \gamma \gamma}\left(-\mathbf{u},-\mathbf{u}_{1}\right) \\
& +\left(\frac{n_{\nu \gamma}^{2}}{n_{\nu \alpha}^{2}}\right) \Pi_{\gamma} \Sigma_{\nu \gamma \alpha}(\mathbf{u}) p_{\nu \gamma \alpha}\left(-\mathbf{u},-\mathbf{u}_{1}\right)
\end{aligned}
$$

As in the OST case, the expression of the different terms of the right side of Eq.39, associated with the use of time reversal conditions, can be deduced from Appendix B.

\section{Radiative Fourier law}

The radiative flux within a non-Beerian effective phase of an OT, OST or ST2 medium can be modelled by a radiative Fourier law in conditions such that the GRTE 
degenerates into a RTE, under two strong validity conditions detailed in Sec.5.1. This radiative Fourier law is then obtained by a perturbation method, similar to the Chapman-Enskog theory[44] based on the Boltzmann equation. This method has been developed for obtaining analytic general expressions of the scalar radiative conductivity $k^{R}(T)$ in the case of an isotropic OT Beerian medium, with interfacial gray or non-gray reflection law[34] and also by Chalhafi et al.[25] for the non-Beerian effective phase of a strongly anisotropic OT medium. As shown in Sec.4.4.3, it can be applied to a non-Beerian effective phase of an OST medium only if the transparent fluid phase and the interfaces have the same temperature field, as a global RTE with two sources at different teperatures is not valid.

Section 5.2 deals with an original development of the perturbation model in the case of non-Beerian effective phases of a strongly anisotropic ST2 medium. A radiative Fourier law is never valid in STT case, as shown in Sec.5.3.

\subsection{Fourier law validity conditions}

The validity conditions of the radiative Fourier law are the same for a RTE issued from the degeneration of a GRTE of a non-Beerian effective phase and for a RTE associated with a Beerian medium. These validity conditions[46] are here simply summarised in the case of an isotropic semi-transparent Beerian medium.

The scattering phase function of such a medium only depends on the scattering angle cosine $\mu=\mathbf{u}_{1} \cdot \mathbf{u}$ and scattering is globally characterised by the scattering asymmetry factor $g[47,48]$ defined by

$$
g_{\nu}=\frac{1}{2} \int_{-1}^{1} p_{\nu}(\mu) \mu \mathrm{d} \mu .
$$

Radiative transfer is characterised by a radiative Fourier law, i.e. a scalar radiative conductivity $k^{R}$, within a possible region of the medium that fulfils the three following conditions [46]:

i) This region is homogeneous;

ii) The distances between all the points of this region and the porous medium boundaries are optically thick. Indeed, in the zone that does not fulfil this condition 
the radiative flux is strongly influenced by the boundary radiative conditions (black or reflecting opaque walls for instance). This last zone is called in the following radiative boundary layer.

iii) For a given relative discrepancy $\zeta$ over the radiative power, the temperature field fulfils, at any point of the region, the inequality[46]

$$
\frac{1}{T} \frac{\mathrm{d} T}{\mathrm{~d} x}<\chi(\zeta) \kappa^{e f f}(\omega, g),
$$

where $\kappa^{\text {eff }}(\omega, g)$ is an effective absorption coefficient that takes into account possible multiple scattering events along the paths of the rays and depends on the albedo $\omega$ and on the scattering asymmetry factor $g$.

The radiative conductivity is due to a large number of successive emission-absorption sequences along short effective distances, often shortened by multiple scattering events[46]. It is physically ruled by an effective absorption coefficient and not by an extinction coefficient. For instance, the value of the generalised extinction coefficient, only due to scattering, can be extremely large within the effective phase associated with a real transparent phase of a STT medium, but the Fourier law is never valid within this phase as there is no absorption, and consequently no emission. It is also not valid within the other absorbing phase, as the transparent phase generates a long range connection with other regions of the medium, as seen in Sec.5.3.

The functions $\chi(\zeta)$ and $\kappa^{e f f}(\omega, g)$ are tabulated in Ref.[46]. For instance, for $\zeta=$ $10^{-2}, \chi$ is equal to 0.033 . If the albedo is equal to 0.5 and $g$ to -0.3 , typical values of backscattering by a porous medium, the criterion corresponds to $\kappa^{e f f}=1.70 \kappa$. For a typical pore size of $100 \mu \mathrm{m}$ and a $\kappa$ value of $50 \mathrm{~cm}^{-1}$, the radiative Fourier law is valid if: $\frac{\mathrm{d} T}{\mathrm{~d} x}<2.8 \mathrm{TK} / \mathrm{cm}$. This criterion is generally valid at high temperature. For instance, at $2000 \mathrm{~K}$, the temperature variation has to be smaller than $56 \mathrm{~K}$ for this pore size of $100 \mu \mathrm{m}$ and, at $500 \mathrm{~K}$, smaller than $14 \mathrm{~K}$.

\subsection{Fourier law for a ST2 medium}

If the two phases of a ST2 medium are characterised by different temperature fields, Fourier laws are not valid, even if these phases are Beerian, due to the prepon- 
derant exchanges between the two phases along distances of small optical thicknesses. If the temperature fields of the non-Beerian effective phases of a ST2 medium are identical and if the validity conditions of Sec.5.1 are fulfilled, radiative transfer is ruled by a unique RTE (Eq.39) and a radiative Fourier laws is valid for the whole medium, as developed in the following.

Consider the effective phases of a ST2 medium that are practically isothermal at the unique temperature $T(M)$ and are assumed to be optically thick within a sphere of radius $\delta$ around a point $M ; \delta$ is typically larger than five times $1 / \kappa_{\text {eff }}$ the effective mean free path of photons between their emission and their absorption, after possible multiple scattering events (see Eq.42). The position of any point $M$ is now defined at macroscale, i.e. at a spatial scale larger than $\delta$, by $\mathbf{R}$ or by $(\mathbf{u}, S)$ where $S$ is the abscissa along the axis $\mathbf{u}$. The aim of this Section is to express the radiative flux vector at macro-scale $\mathbf{q}^{R}(\mathbf{R})$ given by Eq.3 vs the intensity field at macroscale $I_{\nu}(\mathbf{u}, \mathbf{R})$. By analogy with the conduction case, a radiative Knudsen number is introduced

$$
\mathrm{Kn}_{\nu}^{R}(\mathbf{u})=\frac{1}{B_{\nu}(\mathbf{u}) \delta}<<1
$$

It is based on $B_{\nu}(\mathbf{u})$ instead of $\kappa_{e f f}$, which would be more physical, for the sake of simplicity, by assuming that the value of the effective absorption coefficient is large; Anyway, $B_{\nu}(\mathbf{u})$ will disappear in the following. By setting: $\mathbf{R}^{+}=\mathbf{R} / \delta$ and $S^{+}=S / \delta$, the RTE (Eq.39), which is in fact a Boltzmann equation applied to photon momentum[48], becomes

$$
\begin{aligned}
I_{\nu}\left(\mathbf{u}, \mathbf{R}^{+}\right) & =-\operatorname{Kn}_{\nu}^{R}(\mathbf{u}) \mathbf{u} \cdot \operatorname{grad}_{\mathbf{R}}^{+}\left[I_{\nu}\left(\mathbf{u}, \mathbf{R}^{+}\right)\right]+\frac{\kappa_{\nu}}{B_{\nu}(\mathbf{u})} n_{\nu}^{2} I_{\nu}^{\circ}\left[T\left(\mathbf{R}^{+}\right)\right] \\
& +\frac{\sigma_{\nu}}{4 \pi B_{\nu}(\mathbf{u})} \int_{0}^{4 \pi} p_{\nu}\left(\mathbf{u}_{1}, \mathbf{u}\right) I_{\nu}\left[\mathbf{u}_{1}, R_{1}^{+}\left(\mathbf{u}_{1}\right)\right] \mathrm{d} \Omega\left(\mathbf{u}_{1}\right) \\
& +\frac{\Sigma_{\nu i}(\mathbf{u})}{4 \pi B_{\nu}(\mathbf{u})} \int_{0}^{4 \pi} p_{\nu i}\left(-\mathbf{u},-\mathbf{u}_{1}\right) I_{\nu}\left[\mathbf{u}_{1}, R_{1}^{+}\left(\mathbf{u}_{1}\right)\right] \mathrm{d} \Omega\left(-\mathbf{u}_{1}\right) .
\end{aligned}
$$

Equation 44 is then solved, as in the Chapman Enskog model, by a perturbation method, of perturbation parameter $\operatorname{Kn}_{\nu}^{R}(\mathbf{u})$. At the perturbation order 0 , the first 
term of the right side of Eq.44, transport term, which is negligible compared to the other terms, is not accounted for. The corresponding solution $I_{\nu}^{(0)}$ is the equilibrium intensity $n_{\nu}^{2} I_{\nu}^{\circ}\left[T\left(\mathbf{R}^{+}\right)\right]$. But the flux associated with this isotropic intensity is zero. It is then assumed that the solution expressed in dimensional quantities writes at macroscale

$$
I_{\nu}(\mathbf{u}, \mathbf{R})=I_{\nu}^{(0)}[T(\mathbf{R})]+I_{\nu}^{(1)}(\mathbf{u}, \mathbf{R}) \quad \text { with }: \quad \frac{I_{\nu}^{(1)}(\mathbf{u}, \mathbf{R})}{n_{\nu}^{2} I_{\nu}^{\circ}[T(\mathbf{R})]}<<1 .
$$

$I_{\nu}^{(1)}(\mathbf{u}, \mathbf{R})$ is anisotropic and generates the radiative flux. It is solution, at the perturbation order 1, of the implicit equation obtained by removing in Eq.44 the emission term, of perturbation order 0 , and replacing $I_{\nu}(\mathbf{u}, \mathbf{R})$ in the transport term with the solution at the perturbation order zero. By introducing the linear functional

$$
\begin{aligned}
\mathcal{L}_{\nu}\left[X\left(\mathbf{u}_{1}\right)\right](\mathbf{u}) & =\frac{\sigma_{\nu}}{4 \pi B_{\nu}(\mathbf{u})} \int_{0}^{4 \pi} p_{\nu}\left(\mathbf{u}_{1}, \mathbf{u}\right) X\left(\mathbf{u}_{1}\right) \mathrm{d} \Omega\left(\mathbf{u}_{1}\right) \\
& +\frac{\Sigma_{\nu i}(\mathbf{u})}{4 \pi B_{\nu}(\mathbf{u})} \int_{0}^{4 \pi} p_{\nu i}\left(-\mathbf{u},-\mathbf{u}_{1}\right) X\left(\mathbf{u}_{1}\right) \mathrm{d} \Omega\left(-\mathbf{u}_{1}\right)
\end{aligned}
$$

and setting $C(\mathbf{u}, \nu, T)=\left[n_{\nu}^{2} / B\left(\mathbf{u}_{1}\right)\right] \mathrm{d} I_{\nu}^{\circ}(T) / \mathrm{d} T$, the solution $I_{\nu}^{(1)}$ is obtained by an iterative approach

$$
I_{\nu}^{(1)}(\mathbf{u})=-C(\mathbf{u}, \nu, T) \mathbf{u} \cdot \operatorname{grad}_{\mathbf{R}} T-\sum_{k=1}^{\infty} \mathcal{L}_{\nu}^{k}\left[C\left(\mathbf{u}_{1}, \nu, T\right) \mathbf{u} \cdot \operatorname{grad}_{\mathbf{R}} T\right](\mathbf{u})
$$

where $k$ is the iteration order. Consequently, the final solution $I_{\nu}^{(1)}(\mathbf{u}, \mathbf{R})$ is proportional to $-\mathbf{u} \cdot \operatorname{grad}_{\mathbf{R}} T$. Applied to Eq.3 it leads to a radiative Fourier law, i.e. in tensorial notation by using the Cartesian coordinates $X_{l}$ at macroscale

$$
q_{m}^{R}\left(X_{l}\right)=-k_{m n}^{R}\left[T\left(X_{l}\right)\right] \quad \frac{\partial}{\partial X_{n}} T\left(X_{l}\right)
$$




\subsection{No Fourier law for a STT medium}

The effective phase of a STT medium that is associated with a transparent real phase is never optically thick. Consequently a radiative Fourier law is never valid for the two effective phases of this porous medium, even if they are Beerian.

An illustration of the limits of the Fourier law is given in Fig.5 where the results
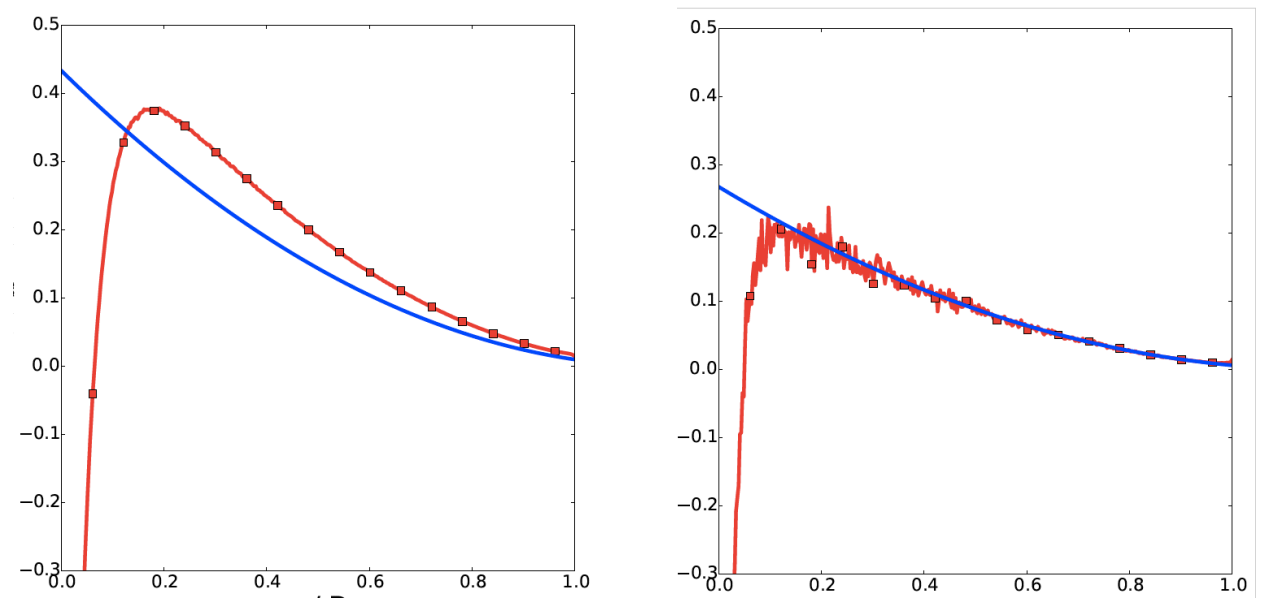

Figure 5: Normalised radiative power field $\mathcal{P}^{R} L / \sigma T(0)^{4}(x / L)$ obtained from the GRTE and the radiative Fourier law applied to a layer of insulation felt of absorbing fibres, of thickness $L ; T(0)=$ $2000 \mathrm{~K}, T(L)=300 \mathrm{~K} ; \quad$ Left: $\kappa_{\alpha} d=0.3$; Right: $\kappa_{\alpha} d=100$ (opaque fibres).[31]

associated with a GRTE model are compared with results obtained by a non-physical Fourier model for an insulation felt. The fibres are made of overlapping absorbing cylinders of finite length $L$,diameter $d$ and absorption coefficient $\kappa_{\alpha}$ within a transparent phase. Details are given in Ref.[31]. Two extreme conditions are considered: a) Optically thin fibres; b) Opaque fibres. The results of intermediate fibre optical thicknesses vary between the two presented curves.

Different conclusion can be drawn: i) Whatever the optical thickness of the fibre, the radiative Fourier model leads to strongly erroneous results, in the vicinity of the hot wall; ii) It is not the case at the vicinity of the cold wall, where the weak relative temperature difference along a large region allows a direct linearisation of the flux (trivial case); iii) The Fourier model is valid in the core of the felt as predicted for an OT case, i.e. a STT medium with $\kappa_{\alpha} d=100$, as shown in Fig.5 b; iv) It is never 
valid in the STT case, as shown In Fig.5 a, despite the fact that the absorbing phase is optically thick at the considered scale, as previously discussed in Sec.5.1.

\section{Spatial resolution of the statistical approach}

The spatial resolution of the statistical model applied to radiative transfer is here discussed, both in the core of a porous medium and in its radiative boundary layer (see definition in Sec.5.1), according to the model in use (homogeneous or nonhomogeneous effective phase, transfer model based on a GRTE or on a radiative Fourier law). This discussion is based on:

i) The four spatial scales that are involved in the characterisation of the radiative statistical properties of an effective phase:

- $d_{p}$, the typical pore size;

- $d_{G}$, the spatial resolution of $G_{\text {ext } \alpha}^{t}$ that is determined by a Monte Carlo method. It is only limited by the experimental resolution of a tomography or by the numerical resolution if the medium is theoretically defined. It is generally much smaller than the typical pore size $d_{p}$;

- $d_{s}$, the scale of the shooting zone, of volume $V$ around a point $M$, from which are issued the rays that allow the statistical radiative functions to be built[20, 26, 29, 31], whatever the types of medium (OT, OST, STT or ST2), of effective phase (Beerian or non-Beerian) and of interfacial transmission and reflection laws. This zone has to be representative of the porous medium and as small as possible;

- $d_{\text {ext }}$, the typical size of the extinction zone of the shot rays, which is generally larger than the shooting zone. The extinction zone is identical to the shooting zone when all the boundaries of this last zone are opaque or characterised by a total specular reflection of the rays that is associated with the symmetries of a porous medium of regular morphology;

ii) $d_{T}$, the spatial resolution of the temperature field that depends on the coupling of the different modes of heat transfer;

iii) $\delta$, the typical spatial scale of the radiative boundary layer. 


\subsection{Homogeneous effective phase}

Some typical examples allow the common case of radiative transfer within a homogeneous effective phase to be illustrated:
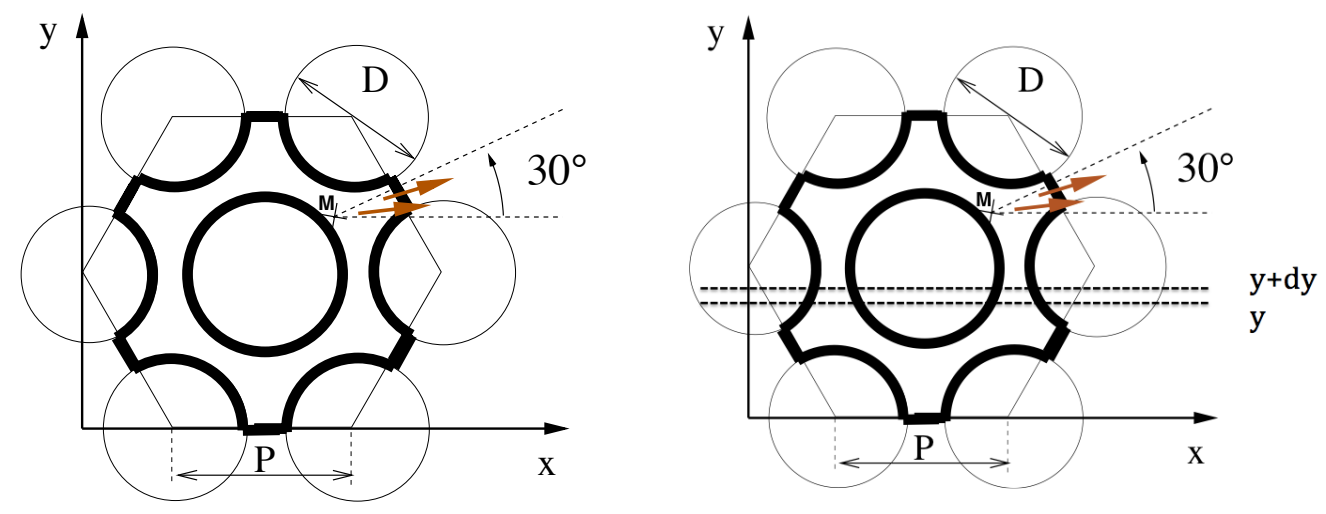

Figure 6: a) Left: Cross-section normal to the axis $z$ of a bundle of parallel rods[25, 29]; Triangular configuration; $\theta$, angle with the axis $z ; \varphi$, azimuth defined by reference to the axis $x$. Merged shooting and extinction zones enclosed between the bold lines; Shot directions included between the dashed lines within a $30^{\circ} \varphi$ angle range. A perfect specular reflection $(\rho=1)$ is applied at any impact over a symmetry plane, within the extinction zone[25, 29]. b) Right: Cross section of the arbitrary thin shooting zone $y, y+\mathrm{d} y$ when the flux vector is parallel to the axis $y$. (EM2C/IRSN study)

i) A statistically homogeneous porous medium is often periodic or practically periodic. The merged shooting and extinction zones are then defined both by the periodic structure and the set of the medium symmetries. It is the case for instance of the bundle of rods shown in Fig.6 a: The shooting and extinction zones are a volume, of hight $\delta z$ and of scale $d_{s}$, that is limited by the bold lines within a cross section normal to the opaque rods.

Even if the spatial resolution $d_{G}$ of $G_{\text {ext } \alpha}$ is much smaller than the pore size $d_{p}$, the rays that contribute to $G_{e x t \alpha}$ are averaged over this shooting and extinction volume of scale $d_{r}$, which is of the same order of magnitude as the pore size $d_{p}$, i.e.

$$
d_{G}<<d_{T}<<d_{s}=d_{e x t} \simeq d_{p} .
$$

The spatial resolution of radiative transfer results, based on a RTE or a GRTE for a Beerian or a non-Beerian effective phase respectively, is then limited by $d_{s}$ in this 
homogeneous approach, even if the spatial resolution $d_{T}$ of the temperature field issued from other heat transfer modes is smaller than $d_{s}$. The homogeneous model is then not optimal, in particular at the vicinity of the boundaries of the porous medium which are often characterised by strong gradients of the temperature field. On the other hand, if the validity conditions of the radiative Fourier law are fulfilled, the medium is practically isothermal within zones larger than $d_{p}$. The spatial resolution of the temperature $d_{T}$ is then larger than $d_{r}$, i.e.

$$
d_{G}<<<d_{s}=d_{e x t} \simeq d_{p}<d_{T} .
$$

The homogeneous model is then pertinent but with a limited spatial resolution.

Moreover, the Fourier model is not valid within the radiative boundary layer of thickness $\delta$ and can lead to absurd results (see for instance Fig.5) .

ii) Some statistically homogeneous porous media do not present any symmetry (case of a foam, DOOS or DOTS of Sec.3.1.1 for instance). If a compact shooting zone is used, for instance a cube, the spatial scales associated with this medium generally verify the conditions

$$
d_{G}<<d_{p} \simeq d_{T}<<d_{s}<<d_{e x t} .
$$

as the the typical size $d_{s}$ of the shooting zone is equal to some typical pore sizes $d_{p}$. The practical spatial resolution of radiative transfer, based on a RTE or a GRTE, for a Beerian or a non-Beerian effective phase respectively, is then limited by $d_{s}$, which a priori is larger than the spatial resolution $d_{T}$ of the temperature field that is assumed to be three-dimensional. As in case i) this modelling is not optimal. A model based on a radiative Fourier also presents the same limitation as in case i).

iii) In practical applications, the statistically homogeneous media of the paragraph ii) often present two dimensions, along $x$ and $y$, that are much larger than the dimension along $z$ and are often practically isothermal. The radiative flux vector is then practically orthogonal to the directions $x$ and $y$ that are assumed to be infinite. 
In the core of the porous medium, the pertinent shooting zone is then a thin slice that is representative of the medium. The following conditions are verified

$$
d_{G}<<d_{s} \simeq d_{T}<<d_{p}<<d_{e x t} \simeq \delta
$$

A homogeneous approach based on a GRTE is accurate as $d_{s}$ and $d_{T}$ are of the same order of magnitude. In the modelling, the extinction cumulative distribution functions that are associated with directions $\mathbf{u}$ oriented towards the boundaries are simply truncated at the boundaries as the extinction scale $d_{e x t}$ is much larger than $d_{s}$.

A radiative Fourier law can also be used as previously in the core of the porous medium.

\subsection{Non-homogeneous effective phase}

Consider now non-homogeneous effective phases. If the temperature field is onedimensional along an axis $y$, a periodic porous medium as the rod bundle of case i) can be characterised by using a very thin shooting zone, a slice between $y$ and $y+\delta y$, that is practically infinite along $x$ and $z$, as shown in Fig. 6 b. As the medium is statistically homogeneous along $x$ and $z$, the porosity $\Pi(y)$ of the shooting zone is accurately defined. It is characterised by strong variations along $y$ between a minimum value and unity. This last value corresponds to a transparent medium. A non-homogeneouse model of radiative transfer, defined in Sec.3.4 and necessary based on a GRTE, can be applied in these conditions. Moreover, it also allows radiative transfer within the radiative boundary layer to be accurately modelled, even if the scale $d_{\text {ext }}$ of the extinction zone is much larger than the thickness $\delta y$ of the shooting zone. Specific radiative statistical functions are then associated with any elementary shooting zone.

\section{Conclusion}

This study deals with radiative transfer within macroporous media with Opaque and Transparent or Semi-Transparent phases (OT or OST), with Semi-Transparent 
and Transparent phases (STT) or with two Semi-Transparent phases (ST2), in which the geometrical optics laws are assumed to be valid. It is focused on media that present after homogenisation non-Beerian effective phases such that extinction is not characterised by an exponential law. When the medium is statistically anisotropic, a directional characterisation of the radiative properties is necessary, as the radiative flux vector then generally presents preferential directions in radiative transfer applications.

Within a non-Beerian effective phase of an OST, a STT or a ST2 medium a strong correlation occurs between transmission, from any emission or scattering source point $M$ of the real semi-transparent phase to an interfacial impact point $M^{\prime}$, and interfacial extinction by scattering or absorption at $M^{\prime}$. An interfacial extinction cumulative distribution function, internal and external scattering cumulative probabilities and associated scattering phase functions exhaustively characterise an effective phase of a STT or ST2 medium. The external scattering cumulative probability is replaced with an interfacial absorption cumulative probability in the case of an OST medium. Moreover, it has been shown from the reciprocity theorem that, within an effective phase of a OT or OST medium, interfacial emission at a point $M$ is also strongly correlated with interfacial transmission from $M$ to another point $M^{\prime}$ and interfacial extinction by scattering or absorption at $M^{\prime}$. The associated extinction cumulative distribution function, absorption and internal scattering cumulative probabilities and internal scattering phase function are then correlated with the interfacial emission source term.

If reflection or transmission is diffuse, i.e. independent of both the incidence and final directions, an internally or externally scattered intensity at $M^{\prime}$ is correlated neither with the incident intensity at $M^{\prime}$ nor with the following transmission and extinction phenomena, even if the effective phase is non-Beerian. The radiative statistical functions of this effective phase are then unique.

In these conditions, a unique Generalised Radiative Transfer Equation (GRTE) is associated with any non-Beerian effective phase of an OT, OST, STT or ST2 medium, instead of a classical Radiative Transfer Equation (RTE). It is directly expressed in terms of the previously defined radiative statistical functions instead of extinction, 
scattering and absorption coefficients and takes into account the radiative boundary conditions. A GRTE can be easily solved by any classical Monte Carlo radiative transfer model, as it is directly expressed in terms of cumulative distribution functions, which is the requirement of a Monte Carlo approach.

Under specific validity conditions the GRTE of a homogeneous effective propagation phase of an OT medium degenerates into a classical RTE and a radiative Fourier can be applied within this effective phase. In the case OST or ST2 medium, this model can only be applied if the homogeneous effective phases are characterised by the same temperature field. A radiative Fourier is never valid in the cases of homogeneous effective phases of a STT medium or of a non-homogeneous effective phase of any type.

If reflection or transmission is non-diffuse, as in the case of a Fresnel-Descartes law, any scattering source term within a non-Beerian effective phase is strongly correlated with the complete set of foregoing successive events: Possible interfacial emission and, in any case, all successive interfacial scattering events. Instead of a unique GRTE, the effective phase is then characterised by a set of specific GRTEs associated with all the successive emission and scattering source terms. Any specific GRTE is expressed in terms of specific radiative statistical functions, which are strongly correlated with all the corresponding radiative functions associated with the foregoing events. This more complex problem can also been solved by a Monte Carlo radiative transfer model.

A non-homogeneous effective phase, with strong volume fraction gradients, is characterised by radiative statistical functions that also depend on the coordinates of the source points. All the previous models of radiative properties are generalised by using a global transmissivity, product of the volume fraction at the source point by the transmissivity deduced from the extinction cumulative distribution function. The method can easily be applied to media with two dimensions that are assumed to be infinite.

The spatial limitations of the transfer methods based, on one hand, on a GRTE or a radiative Fourier law and, on the other one, on a homogeneous or a nonhomogeneous approach have been discussed from the comparison of all the scales 
associated with the resolution of the radiative statistical functions, the pore sizes, the shooting and extinction zones of the numerical approach, the resolution of the temperature fields and the width of the radiative boundary layer.

\section{Aknowledgments}

The authors acknowledge for their determinant contributions to the statistical modelling of radiative properties of porous media Drs Dominique Baillis, Fabien Bellet, Miloud Chahlafi, Elie Chalopin, Yann Dauvois, Florian Fichot, Hector Gomart, Estelle Iacona, Manuel Tancrez, Marie Zarrouati, and Barbar Zhegondy. They acknowledge the financial supports of Air Liquide, Commissariat aux Énergies Alternatives (CEA/DAM), CNRS, Gaz de France, Institut de Radioprotection et Sûreté Nucléaire and of the Ministère de la Recherche.

\section{Appendix A. Interfacial shots and volume shots}

\section{A.1. General case}

Are $G_{\text {ext } \alpha}^{S t}$ associated with interfacial source points and $G_{\text {ext } \alpha}^{t}$ associated with volume source points, defined in Sec.3.1.1, and consequently $G_{e x t \alpha}^{S}$ and $G_{\text {ext } \alpha}$ identical or different?

In $G_{\text {ext } \alpha}^{S t}(\mathbf{u}, w), w$ is the length of the chord between an interfacial source point $D(s)$ and an interfacial extinction one $E\left(s^{\prime}\right) . G_{e x t \alpha}^{S t}(\mathbf{u}, w)$ is in fact the cumulative distribution function of the chords $D E$ of length $w ; 1-G_{\text {ext } \alpha}^{S t}(\mathbf{u}, w)$ is then the probability that the length of a chord $D E$ is larger than $w$. Int he case of a phase that presents only convex interfaces, for instance the space outside non-overlapping cylinders or spheres, $w$ varies between a non-zero minimum length $w_{\min }$ and a maximum one $w_{\max }$, possibly infinite.

On the other hand, $G_{e x t \alpha}^{t}(\mathbf{u}, w)$ is the cumulative distribution function of the segments $M E$ of length $w$ joining a volume point of the phase $M(s)$ to an interfacial impact point $E\left(s^{\prime}\right)$ and $F_{\text {ext } \alpha}^{t}(\mathbf{u}, w) \mathrm{d} w$ is the probability that the length of a segment $M E$ belongs to the range $[w, w+\mathrm{d} w]$, where $F_{\text {ext } \alpha}^{t}(\mathbf{u}, w)$ is the associated distribution function. Now $w$ varies between 0 and a maximum length $w_{\max }$, possibly infinite.

At any segment of impact point $E$ in the direction $\mathbf{u}$ and length $w$, is associated a unique chord of same impact point $E$ and direction $\mathbf{u}$ and of length larger than $w$. As the density of points in any element of length $\mathrm{d} w$ is uniform, the number of these segments $M E$ of direction $\mathbf{u}$ and of length in the range $[w, w+\mathrm{d} w]$ larger than $w_{\text {min }}$ is then proportional 
to the number of chords $D E$ of lengths larger than $w$ : If $w>w_{\min }, 1-G_{\text {ext } \alpha}^{S t}(\mathbf{u}, w)$ is proportional to $F_{\text {ext } \alpha}^{t}(\mathbf{u}, w)$. Consequently, $G_{\text {ext } \alpha}^{S t}(\mathbf{u}, w)$ verifies the equations

$$
w<w_{\text {min }}: 1-G_{\text {ext } \alpha}^{S t}(\mathbf{u}, w)=1, \quad w>w_{\text {min }}: 1-G_{\text {ext } \alpha}^{S t}(\mathbf{u}, w)=\frac{F_{\text {ext } \alpha}^{t}(\mathbf{u}, w)}{F_{\text {ext } \alpha}^{t}\left(\mathbf{u}, w_{\text {min }}\right)} .
$$

Indeed $1-G_{\text {ext } \alpha}^{S t}(\mathbf{u}, w)$ is equal to 1 for $w=w_{\text {min }}$.

For a transparent effective phase, $G_{\text {ext } \alpha}^{S t}$ associated with interfacial source points is finally linked to $G_{\text {ext } \alpha}^{t}$ associated with volume source points by

$$
\begin{array}{ll}
w<w_{\text {min }}: & 1-G_{\text {ext } \alpha}^{S t}(\mathbf{u}, w)=1 \\
w>w_{\text {min }}: & 1-G_{\text {ext } \alpha}^{S t}(\mathbf{u}, w)=\frac{\mathrm{d}}{\mathrm{d} w} G_{\text {ext } \alpha}^{t}(\mathbf{u}, w) /\left(\frac{\mathrm{d}}{\mathrm{d} w} G_{\text {ext } \alpha}^{t}(\mathbf{u}, w)\right)_{w_{\text {min }}} .
\end{array}
$$

Equation A.2 generalises the result of Ref.[38], valid for a phase that presents concave interfaces, such that: $w_{\min }=0$.

\section{A.2. Beerian case}

For an effective phase characterised by $w_{\min }>0,1-G_{\text {ext } \alpha}^{S t}(\mathbf{u}, w)$ is equal to 1 for $w<w_{\text {min }}$, which is not a Beerian behaviour.

On the contrary, in the case of an effective phase characterised by $w_{\min }=0,1-$ $G_{\text {ext } \alpha}^{t}(\mathbf{u}, w)$ and $1-G_{\text {ext } \alpha}^{S t}(\mathbf{u}, w)$ have the same expression $\exp \left[-\beta_{\nu}(\mathbf{u}) w\right]$ if this effective phase is Beerian, as shown by Eq.A.2. This property has often been used, without justification[20, 22, 26, 27, 29], etc.

\section{Appendix B. Some properties of scattering}

As discussed in Sec.4.4, coupled RTEs are not valid for a ST2 medium characterised by two optically thick and practically isothermal non-Beerian effective phases if their temperatures differ. Nevertheless, if the two non-Beerian effective phases are at the same temperature, the whole medium can be characterised, in these conditions, by a global RTE built by summing the two individual RTEs. Such an individual RTE, which also 
corresponds to a possibly Beerian interfacial behaviour, writes

$$
\begin{aligned}
& \frac{\mathrm{d}}{\mathrm{d} s} I_{\nu j}(\mathbf{u}, s)=-\left[\beta_{\nu m_{j}}+\sigma_{\nu j j}(\mathbf{u})+\sigma_{\nu j k}(\mathbf{u})\right] I_{\nu j}(\mathbf{u}, s)+\kappa_{\nu m_{j}} \Pi n_{\nu j}^{2} I_{\nu}^{\circ}[T(s)] \\
& +\int_{0}^{4 \pi} \sigma_{\nu j j}\left(\mathbf{u}_{1}\right) \frac{p_{\nu j j}\left(\mathbf{u}_{1}, \mathbf{u}\right)}{4 \pi} I_{\nu j}\left(\mathbf{u}_{1}, s_{1}\right) \mathrm{d} \Omega_{j 1}\left(\mathbf{u}_{1}\right)+\int_{0}^{4 \pi} \sigma_{\nu k j}\left(\mathbf{u}_{1}\right) \frac{p_{\nu k j}\left(\mathbf{u}_{1}, \mathbf{u}\right)}{4 \pi} I_{\nu k}\left(\mathbf{u}_{1}, s_{1}\right) \mathrm{d} \Omega_{k 1}\left(\mathbf{u}_{1}\right), \\
& +\sigma_{\nu m_{j}} \int_{0}^{4 \pi} \frac{p_{\nu m_{j}}\left(\mathbf{u}_{1} \cdot \mathbf{u}\right)}{4 \pi} I_{\nu j}\left(\mathbf{u}_{1}, s_{1}\right) \mathrm{d} \Omega_{j 1}\left(\mathbf{u}_{1}\right) .
\end{aligned}
$$

In Ideal Thermal Equilibrium (ITE) at the temperature $T$, the detailed balance principle, or principle of microreversibility[35, 36] states that all types of emission and scattering source terms are equal to the reciprocal absorption and scattering dissipation terms. After simplification, the equality between the source and dissipation terms corresponding to external interfacial scattering writes

$$
\Pi_{j} n_{\nu j}^{2} \sigma_{\nu j k}(\mathbf{u})=\Pi_{k} n_{\nu k}^{2} \int_{0}^{4 \pi} \sigma_{\nu k j}\left(\mathbf{u}_{1}\right) \frac{p_{\nu k j}\left(\mathbf{u}_{1}, \mathbf{u}\right)}{4 \pi} \mathrm{d} \Omega_{k 1}\left(\mathbf{u}_{1}\right)
$$

and, by using the Clausius theorem $[48,49]$ that states that: $n_{\nu k}^{2} \mathrm{~d} \Omega_{k 1}\left(\mathbf{u}_{1}\right)=n_{\nu j}^{2} \mathrm{~d} \Omega_{j 1}\left(\mathbf{u}_{1}\right)$,

$$
\Pi_{j} \sigma_{\nu j k}(\mathbf{u})=\Pi_{k} \int_{0}^{4 \pi} \sigma_{\nu k j}\left(\mathbf{u}_{1}\right) \frac{p_{\nu k j}\left(\mathbf{u}_{1}, \mathbf{u}\right)}{4 \pi} \mathrm{d} \Omega_{j 1}\left(\mathbf{u}_{1}\right)
$$

This last equation is entirely expressed in the phase j. The reciprocity theorem[50] applied, in ITE conditions, to the external scattering of an elementary beam $d \Omega_{k 1}$ by a volume element $\Pi_{k} \mathrm{~d} V$ of the effective phase k towards the volume element $\Pi_{j} \mathrm{~d} V$ of the effective phase $\mathrm{j}$ allows the following equality to be written

$$
\begin{aligned}
& \left(n_{\nu k}^{2} I_{\nu}^{\circ}(T) \mathrm{d} \Omega_{k 1}\left(\mathbf{u}_{1}\right) \Pi_{k} \mathrm{~d} V \mathrm{~d} \nu\right) \sigma_{\nu k j}\left(\mathbf{u}_{1}\right) \frac{p_{\nu k j}\left(\mathbf{u}_{1}, \mathbf{u}\right)}{4 \pi} \mathrm{d} \Omega_{j}(\mathbf{u}) \\
= & \left(n_{\nu j}^{2} I_{\nu}^{\circ}(T) \mathrm{d} \Omega_{j}(-\mathbf{u}) \Pi_{j} \mathrm{~d} V \mathrm{~d} \nu\right) \sigma_{\nu j k}(-\mathbf{u}) \frac{p_{\nu j k}\left(-\mathbf{u},-\mathbf{u}_{1}\right)}{4 \pi} \mathrm{d} \Omega_{k 1}\left(-\mathbf{u}_{1}\right) .
\end{aligned}
$$

By using the Clausius theorem, the equality $\mathrm{d} \Omega_{j}(\mathbf{u})=\mathrm{d} \Omega_{j}(-\mathbf{u})$ and by simplifying Eq.B.4 by $n_{\nu j}^{2} I_{\nu}^{\circ}(T) \mathrm{d} \Omega_{j}(\mathbf{u}) \mathrm{d} V \mathrm{~d} \nu$ this equation becomes

$$
\Pi_{k} \sigma_{\nu k j}\left(\mathbf{u}_{1}\right) \frac{p_{\nu k j}\left(\mathbf{u}_{1}, \mathbf{u}\right)}{4 \pi} \mathrm{d} \Omega_{j 1}\left(\mathbf{u}_{1}\right)=\Pi_{j} \sigma_{\nu j k}(-\mathbf{u}) \frac{p_{\nu j k}\left(-\mathbf{u},-\mathbf{u}_{1}\right)}{4 \pi} \mathrm{d} \Omega_{k 1}\left(-\mathbf{u}_{1}\right)
$$


Equations B.3 and B.5 then lead to

$$
\sigma_{\nu j k}(\mathbf{u})=\sigma_{\nu j k}(-\mathbf{u}) \int_{0}^{4 \pi} \frac{p_{\nu j k}\left(-\mathbf{u},-\mathbf{u}_{1}\right)}{4 \pi} \mathrm{d} \Omega_{k 1}\left(-\mathbf{u}_{1}\right)=\sigma_{\nu j k}(-\mathbf{u})
$$

This result can also be applied to internal scattering:

$$
\sigma_{\nu j j}(\mathbf{u})=\sigma_{\nu j j}(-\mathbf{u})
$$

By using Eq.B.5, the Clausius theorem and Eq.B.6, the elementary external scattering source term becomes

$\sigma_{\nu k j}\left(\mathbf{u}_{1}\right) \frac{p_{\nu k j}\left(\mathbf{u}_{1}, \mathbf{u}\right)}{4 \pi} I_{\nu k}\left(\mathbf{u}_{1}, s_{1}\right) \mathrm{d} \Omega_{k 1}\left(\mathbf{u}_{1}\right)=\left(\frac{n_{\nu j}^{2} \Pi_{j}}{n_{\nu k}^{2} \Pi_{k}}\right) \sigma_{\nu j k}(\mathbf{u}) \frac{p_{\nu j k}\left(-\mathbf{u},-\mathbf{u}_{1}\right)}{4 \pi} I_{\nu k}\left(\mathbf{u}_{1}, s_{1}\right) \mathrm{d} \Omega_{k 1}\left(-\mathbf{u}_{1}\right)$.

Consequently, Equation B.1 also simply writes

$$
\begin{aligned}
& \frac{\mathrm{d}}{\mathrm{d} s} I_{\nu j}(\mathbf{u}, s)=-\left[\beta_{\nu m_{j}}+\sigma_{\nu j j}(\mathbf{u})+\sigma_{\nu j k}(\mathbf{u})\right] I_{\nu j}(\mathbf{u}, s)+\kappa_{\nu m_{j}} \Pi_{j} n_{\nu j}^{2} I_{\nu}^{\circ}[T(s)] \\
& +\sigma_{\nu m_{j}} \int_{0}^{4 \pi} \frac{p_{\nu m_{j}}\left(\mathbf{u}_{1} \cdot \mathbf{u}\right)}{4 \pi} I_{\nu j}\left(\mathbf{u}_{1}, s_{1}\right) \mathrm{d} \Omega_{j 1}\left(\mathbf{u}_{1}\right)+\sigma_{\nu j j}(\mathbf{u}) \int_{0}^{4 \pi} \frac{p_{\nu j j}\left(-\mathbf{u},-\mathbf{u}_{1}\right)}{4 \pi} I_{\nu j}\left(\mathbf{u}_{1}, s_{1}\right) \mathrm{d} \Omega_{j 1}\left(-\mathbf{u}_{1}\right) \\
& +\left(\frac{n_{\nu j}^{2} \Pi_{j}}{n_{\nu k}^{2} \Pi_{k}}\right) \sigma_{\nu j k}(\mathbf{u}) \int_{0}^{4 \pi} \frac{p_{\nu j k}\left(-\mathbf{u},-\mathbf{u}_{1}\right)}{4 \pi} I_{\nu k}\left(\mathbf{u}_{1}, s_{1}\right) \mathrm{d} \Omega_{k 1}\left(-\mathbf{u}_{1}\right) .
\end{aligned}
$$

Equation 37 applied to a Beerian effective phase of an OT medium then also becomes, by following a similar approach,

$\frac{\mathrm{d}}{\mathrm{d} s} I_{\nu}(\mathbf{u}, s)+\beta_{i}(\mathbf{u}) I_{\nu}(\mathbf{u}, s)=\Pi \kappa_{\nu i}(\mathbf{u}) n_{\nu}^{2} I_{\nu}^{\circ}\left[T_{i}(s)\right]+\sigma_{\nu i}(\mathbf{u}) \int_{0}^{4 \pi} \frac{p_{\nu i}\left(-\mathbf{u},-\mathbf{u}_{1}\right)}{4 \pi} I_{\nu}\left(\mathbf{u}_{1}, s_{1}\right) \mathrm{d} \Omega_{1}\left(-\mathbf{u}_{1}\right)$.

\section{References}

[1] D. Baillis and J. F. Sacadura. Thermal radiation properties of dispersed media: theoretical prediction and experimental characterization. J. Quant. Spectr. Radiat. Transfer, 67(5):327-363, 2000.

[2] L. A. Dombrovsky and D. Baillis. Thermal radiation in disperse systems: An engineering approach. Begell House, New York., 2010. 
[3] J.L. Consalvi, B. Porterie, and J.C. Loraud. A formal averaging procedure for radiation heat transfer in particulate media. Int. J. Heat Mass Transfer, 45:2755-2768, 2002.

[4] M. Quintard and S. Whitaker. Transport in ordered and disordered porous media - I: The cellular average and the use of weighting functions. Transport in Porous Media, 14:163-177, 1994.

[5] M. Quintard and S. Whitaker. Transport in ordered and disordered porous media II: Generalized volume averaging. Transport in Porous Media, 14:179-206, 1994.

[6] M. Quintard and S. Whitaker. Transport in ordered and disordered porous media III: Closure and comparison between theory and experiment. Transport in Porous Media, 15:31-49, 1994.

[7] M. Quintard and S. Whitaker. Transport in ordered and disordered porous media IV: Computer generated porous media for three-dimensional systems. Transport in Porous Media, 15:51-70, 1994.

[8] W. Lipinski, J. Petrasch, and S. Haussener. Application of the spatial averaging theorem to radiative heat transfer in two-phase media. J. Quant. Spectr. Radiat. Transfer, 11:253-258, 2010.

[9] W. Lipinski, D. Keene, S. Haussener, and J. Petrasch. Continuum radiative heat transfer in media consisting of optically distinct components in the limit of geometrical optics. J. Quant. Spectr. Radiat. Transfer, 111:2474-2480, 2010.

[10] J. Randrianalisoa and D. Baillis. Radiative properties of densely packed spheres in semitransparent media: A new geometric optics approach. J. Quant. Spectr. Radiat. Transfer, 111:1372-1388, 2010.

[11] J. Randrianalisoa and D. Baillis. Radiative transfer in dispersed media: comparison between homogeneous phase and multiphase approaches. ASME J. Heat Transfer, 132(023405), 2010. 
[12] J. Randrianalisoa, S. Haussener, D. Baillis, and W. Lipiński. Radiative characterization of random fibrous media with long cylindrical fibers: Comparison of single- and multi-RTE approaches. J. Quant. Spectr. Radiat. Transfer, 202:220-232, 2017.

[13] R. Coquard, D. Baillis, and J. Randrianalisoa. Homogeneous phase and multi-phase approaches for modeling radiative transfer in foams. Int. J. Thermal Sci., 50:1648$1663,2011$.

[14] R. Coquard, J. Randrianalisoa, and D. Baillis. Computational prediction of radiative properties of polymer closed-cell foams with random structure. J. Porous Media, 16:137-154, 2013.

[15] A.V. Gusarov. Homogenization of radiation transfer in two-phase media with irregular phase boundaries. Phys. Rev. B, 77(144201):1-14, 2008.

[16] A.V. Gusarov. A model of averaged radiation transfer in two-phase heterogeneous medium. High Temp.-High Press., 47(3):375-389, 2009.

[17] A.V. Gusarov. Model of radiative heat transfer in heterogeneous multiphase media. Phys. Rev. B, 81 (6)(064202):1-9, 2010.

[18] V. Gusarov and I. Smurov. Homogenized models of radiation transfer in multiphase media. In C. Constanda and M.E. Perez, editors, Integral Methods in Science and Engineering, volume 2, pages 183-192. Birkhauser, Boston, 2010.

[19] A.V. Gusarov. Statistical approach to radiative transfer in the heterogeneous media of thin-wall morphology - I: Theory. ASME J. Heat Transfer, 140 (11)(112701), 2018.

[20] B. Zeghondy, E. Iacona, and J. Taine. Determination of the anisotropic radiative properties of a porous material by radiative distribution function identification (RDFI). Int. J. Heat Mass Transfer, 49:2810-2819, 2006.

[21] S. Haussener, W. Lipinski, J. Petrasch, P. Wyss, and A. Steinfeld. Tomographic characterization of a semitransparent-particle packed bed and determination of its thermal radiative properties. ASME J. Heat Transfer, 131(072701):1-7, 2009. 
[22] S. Haussener, P. Coray, W. Lipinski, P. Wyss, and A. Steinfeld. Tomographybased heat and mass transfer characterization of reticulate porous ceramics for hightemperature processing. ASME J. Heat Transfer, 132(023305):1-9, 2010.

[23] H. Scheurer. Data Book PHEBUS FPT1. Issue J-01.09.95 IS.92.49, CEA, Cadarache, France, 1995.

[24] B. Berthet, J. Bonnin, S. Bayle, N. Hanniet, F. Jeury, S. Gaillot, Y. Garnier, C. Martin, M. Laurie, and B. Siri. Phebus- PF-FPT1 Preliminary report. Technical Report IP/97/334, IPSN/DRS/SEA/LERES, October 1997.

[25] M. Chahlafi, F. Bellet, F. Fichot, and J. Taine. Radiative transfer within non-Beerian porous media with semi-transparent and opaque phases in non equilibrium: Application to reflooding of a nuclear reactor. Int. J. Heat Mass Transfer, 55(13-14):3666$3676,2012$.

[26] M. Tancrez and J. Taine. Direct identification of absorption and scattering coefficients and phase function of a porous medium by a Monte Carlo technique. Int. J. Heat Mass Transfer, 47:373-383, 2004.

[27] J. Petrasch, P. Wyss, and A. Steinfeld. Tomography-based Monte Carlo determination of radiative properties of reticulated porous ceramics. J. Quant. Spectr. Radiat. Transfer, 105:180-197, 2007.

[28] S. Haussener, W. Lipinski, P. Wyss, and A. Steinfeld. Tomography-based analysis of radiative transfer in reacting packed beds undergoing a solid-gas thermochemical transformation. ASME J. Heat Transfer, 132(061201):1-7, 2010.

[29] F. Bellet, E. Chalopin, F. Fichot, E. Iacona, and J. Taine. RDFI determination of anisotropic and scattering dependent radiative conductivity tensors in porous media: Application to rod bundles. Int. J. Heat Mass Transfer, 52:1544-1551, 2009.

[30] M. Zarrouati, F. Enguehard, and J. Taine. Radiative transfer within strongly non homogeneous porous media: Application to a slab of packed particles. Int. J. Heat Mass Transfer, 91:936-947, 2015. 
[31] Y. Dauvois, D. Rochais, F. Enguehard, and J. Taine. Statistical radiative modeling of a porous medium with semitransparent and transparent phases: Application to a felt of overlapping fibres. Int. J. Heat Mass Transfer, 106:601-618, 2017.

[32] M. Zarrouati, F. Enguehard, and J. Taine. Statistical characterization of near wall radiative properties of a statistically non homogeneous and anisotropic porous medium. Int. J. Heat Mass Transfer, 67:776-783, 2013.

[33] J. Petrasch, S. Haussener, and W. Lipinski. Discrete vs. continuum scale simulation of radiative transfer in semi transparent two phase media. J. Quant. Spectr. Radiat. Transfer, 112:1450-1459, 2011.

[34] J. Taine, F. Bellet, V. Leroy, and E. Iacona. Generalized radiative transfer equation for porous medium upscaling: Application to the radiative Fourier law. Int. J. Heat Mass Transfer, 53(19-20):4071-4081, 2010.

[35] R.C. Tolman. The principles of statistical mechanics. Phys. Rev., 13:699, 1924.

[36] B. Diu, D. Lederer, and B. Roulet. Physique Statistique. Hermann, Paris, 1996.

[37] S. Torquato and B. Lu. Chord-length distribution function for two-phase random media. Physical Review E, 47(4):2950-2953, 1993.

[38] Y. Dauvois, M. Zarrouati, D. Rochais, F. Enguehard, and J. Taine. Comments over homogenisation scales for interfacial emission and scattering by a divided medium: Beerian and non-Beerian behaviours. Int. J. Heat Mass Transfer, 103:468-477, 2016.

[39] J.R. Howell, Mengüc P., and R. Siegel. Thermal Radiation Heat Transfer. CRC Press-Taylor and Francis, Washington, Sixth edition, 2001.

[40] M. Modest. Radiative Heat Transfer. Academic Press, Third edition, 2013.

[41] X. Jia and R. Williams. A packing algorithm for particles of arbitrary shapes. Powder Technology, 120:175-176, 2001.

[42] X. Jia, M. Gan, R. Williams, and D. Rhodes. Validation of a digital packing algorithm in predicting powder packing densities. Powder Technology, 174:10-13, 2007. 
[43] M. Zarrouati. Modélisation des transferts radiatifs dans des milieux poreux non Beeriens au voisinage des parois: Application au vaporéformage du méthane. $\mathrm{PhD}$ thesis, Chapter 5 (in English): Radiative transport model for axisymmetrical strongly non homogeneous macrostructured scattering media, Ecole Centrale Paris, April 2015.

[44] S. Chapman and T.G. Cowling. The Mathematical Theory of Non-uniform Gases. Cambridge University Press, 1990.

[45] J.H. Ferziger and N. Kaper. Mathematical Theory of Transport Processes in Gases. Elsevier, U.S.A., 1972.

[46] H. Gomart and J. Taine. Validity criterion of the radiative Fourier law for an absorbing and scattering medium. Phys. Rev. E, 83(2):1-8, 2011.

[47] J. Howell, R. Siegel, and P. Menguc. Thermal Radiation Heat Transfer. CRC Press, Washington, 5th edition, 2011.

[48] J. Taine, F. Enguehard, and E. Iacona. Transferts Thermiques. Dunod, Paris, $5^{\text {th }}$ edition, 2014.

[49] M. Born and E. Wolf. Principles of Optics. Pergamon Press, 4th edition, 1970.

[50] J.J. Greffet and M. Nieto-Vesperinas. Field theory for generalized bidirectional reflectivity: Derivation of Helmholtz's reciprocity principle and Kirchhoff's law. J. Opt. Soc. Am., 15:2735-2744, 1998. 